\title{
Modeling Public Opinion Polarization in Group Behavior by Integrating SIRS-Based Information Diffusion Process
}

\author{
Tinggui Chen $\mathbb{D}^{1},{ }^{1}$ Jiawen Shi, ${ }^{2}$ Jianjun Yang $\mathbb{D}^{3},{ }^{3}$ Guodong Cong $\mathbb{D}^{4},{ }^{4}$ and Gongfa Li $\mathbb{C}^{5}$ \\ ${ }^{1}$ School of Statistics and Mathematics, Zhejiang Gongshang University, Hangzhou, China \\ ${ }^{2}$ Business and Tourism Institute, Hangzhou Vocational and Technical College, Hangzhou, China \\ ${ }^{3}$ Department of Computer Science and Information Systems, University of North Georgia, Oakwood, GA, USA \\ ${ }^{4}$ School of Tourism and Urban-Rural Planning, Zhejiang Gongshang University, Hangzhou, China \\ ${ }^{5}$ Hubei Key Laboratory of Mechanical Transmission and Manufacturing Engineering, \\ Wuhan University of Science and Technology, Wuhan, China
}

Correspondence should be addressed to Tinggui Chen; ctgsimon@mail.zjgsu.edu.cn

Received 16 January 2020; Revised 2 May 2020; Accepted 5 August 2020; Published 26 September 2020

Academic Editor: José Manuel Galán

Copyright ( $\odot 2020$ Tinggui Chen et al. This is an open access article distributed under the Creative Commons Attribution License, which permits unrestricted use, distribution, and reproduction in any medium, provided the original work is properly cited.

Currently, China is in the period of social transformation. Such transformation continuously results in high group polarization behaviors, which attracts many attentions. In order to explore the evolutionary mechanism and formation process of group polarization behavior, this paper proposes a group polarization model which is integrated into the Susceptible-Infected-Recovered-Susceptible (SIRS) epidemic model. In this paper, firstly, the SIRS epidemic model and the factors of relationship strength are introduced based on the J-A model (proposed by Jager and Amblard) to enhance the information transmission and interaction among individuals. In addition, the BA network (proposed by Barabasi and Albert) model is used as the agent adjacency model due to its closeness to the real social network structure. After that, the Monte Carlo method is applied to conduct experimental simulation. Subsequently, this paper analyzes the simulation results in threefold: (1) comparison of polarization processes with and without integration of the SIRS epidemic model; (2) adjusting the immune recovery parameter $\gamma$ and the relationship strength $z$ to explore the role of these two parameters in the polarization process; and (3) comparing the polarization effects of different network structures. Through the experiments, we find that BA network is more polarized than small-world network in the same scale. Finally, corresponding measures are proposed to prevent and mitigate the occurrence of group polarization.

\section{Introduction}

Currently, China is in the period of social transformation. Social mass events take place frequently. The consequent extreme behaviors often cause significant harm and loss to the society and people. Jianrong $\mathrm{Yu}$, the director of the Research Center for social problems of Rural Development Institute of Chinese Academy of Social Sciences, made a speech at the Beijing Lawyers Association that, from 1993 through 2006, the number of China's mass events was increased from 8,709 to 90,000 . While from 2007 through 2009, it was more than 90,000. After 2010, it has surged to more than 100,000 per year [1]. Usually, mass events refer to the temporary coupling groups caused by some social contradictions, which are formed by the aggregation of specific groups or unspecified majority people. They express their requests and opinions or vent their dissatisfaction and create influence by means of group behaviors. With the development of the Internet, the characteristics of high interactivity and low cost of the Internet make more mass events grow on the Internet. Mass events are not just limited in China but occur all over the world. In the famous "Killing of George Floyd" [2] on May 25, 2020, in Minneapolis, USA, when a white police officer Derek Chauvin was arresting a 46-year-old African American man George Floyd for his using a $\$ 20$ counterfeit bill, he knelt on Floyd's neck for eight minutes and 46 seconds while Floyd was handcuffed and lying face 
down. Floyd was begging for his life and repeatedly saying "I cannot breathe". However, the officer disregarded Floyd's implore, and finally Floyd was killed. The following days, after videos made by witnesses and security cameras became public, people in Minneapolis began their protests. Soon after, protests turned into parades and the demonstrations led to violence in at least 30 cities across the United States in the aftermath [3]. In June, the impact was escalated to a nationwide movement "Black Lives Matter". It may be the largest movement in U.S. history [4]. This event has aroused wide attention and discussion of a large number of netizens. Someone said that Floyd should not die because he did not straightly threaten the officer's life, and he was just using a counterfeit $\$ 20$ bill then. The opposite opinion stated that Floyd did not follow the officers' commands and he had a criminal past and previous convictions including a violent criminal history. Therefore, it is legal for the officer to knee Floyd and the officer should not be responsible for his death. With the further propagation of the news, netizens' attitudes towards the event have gradually deepened. Two or more opposite remarks in Twitter and Facebook have appeared, meaning that bipolarization or multidimensional polarization phenomenon has formed. The confrontation between these two views is intensified and even leads to the group behavior in reality.

In fact, public opinion polarization has positive effects. For example, it can promote people's thinking and discussion on social issues. However, due to the influence of different interest groups, the information obtained by Internet users is often inflammatory and one-sided. The polarization of public opinion may lead to the formation of irrational and violent public emotions and uncontrollable behavior and encourage social prejudice and social division, endangering the social and political stability and even detonating the negative consequences of ethnic warfare. Thus, the negative role of public opinion polarization needs more attention.

This paper integrates the SIRS epidemic model [5] into the Jager and Amblard model (the J-A model) [6] and proposes an expanded group polarization model integrating the SIRS epidemic model. Through simulation experiments, individuals who participate in group behavior are defined as agent, then the individual types, individual behaviors, group size, group emergence, and so on are also included into the category of network public opinion evolution. Subsequently, the formation and evolution processes of network public opinion are simulated so as to reveal the internal mechanism of opinion polarization and explore the main influence factors of opinion polarization.

The structure of this paper is organized as follows: Section 2 is the literature review in which the shortcomings of the existing research are discussed. In addition, the contributions of this study are also proposed. Section 3 proposes an opinion polarization model with SIRS epidemic model. Section 4 compares the polarization process with and without integration of SIRS epidemic model. Section 5 is the analysis and discussion of simulation results. Finally, section 6 concludes the paper and proposes future research directions.

\section{Literature Review}

According to the analysis of the existing literature, this section discusses the relevant literatures mainly from the following two aspects: information transmission and public opinion polarization.

In terms of the information transmission, the use of infectious disease dynamics to study network public opinion communication has been relatively mature. For example, Kyrychko and Blyuss [7] derived and studied a time-delayed Susceptible-Infected-Recovered (SIR) model with a general incidence rate. The time delay represented a temporary immunity period, i.e., time from recovery to becoming susceptible again. In the study, both trivial and endemic equilibria were found, and their stability was also investigated. Furthermore, by establishing the Infected-Susceptible-Infected-Susceptible-Recovered (I2S2R) model, Tanimoto [8] introduced evolutionary game theory into epidemiology and regarded vaccination as a game in complex social networks. Zhang and Zhu [9] studied two rumor propagation dynamics with secondary relations and concluded that the propagation intensity of secondary rumors depended on the propagation intensity of initial rumors. Huo and Song [10] studied the interaction between rumors and scientific information and found that rumors and scientific information coexisted under certain transmission probability. Wu and Gergely [11] proposed a new Susceptible-Exposed-Infected-Resistant (SEIR) model, in which infection time depended on the distribution of infection age and had infinite delay. Unlike the research mentioned above, Chen et al. [12] explored the network topology of group event propagation and used artificial immune algorithm to get the network structure characteristics of promoting or inhibiting group synchronous behavior propagation. Boccara and Cheong [13] studied the SIR model of automation network for infectious disease transmission. It was shown that the occurrence of the epidemic in one population might be triggered by the occurrence of the epidemic in the other population. The emphasis was on the influence of the degree of mixing of the individuals which followed from their diffusive motion. In particular, the asymptotic behaviors for very small and very large mixing were determined. However, few scholars have combined the infectious disease model with the process of public opinion polarization. Especially, the state of infected individuals is taken as an intermediate variable to measure whether the individual's attitude changes. Kabir et al. [14] believed that information awareness about contagious diseases had an influential effect on an individual's decision to suppress the diffusion of infections and thus proposed a dynamic system of Susceptible-Infected-Recovered (SIR) with Unaware-Aware (UA) process (SIR-UA). Individuals in this system could be classified into six states as unaware susceptible (SU), aware susceptible (SA), unaware infected (IU), aware infected (IA), unaware recovered (RU), and aware recovered (RA). Furthermore, Kabir et al. [15] constructed a two-layer SIR-UA epidemic model with information transmission and disease transmission and analyzed the impact of different heterogeneous networks on the 
population. In addition, Kabir et al. [16-18] also combined SIR-UA epidemic model with the immune game and considered the impact of different strategy updating rules on the final spread of infectious diseases. Different from these research, our study combines the SIRS epidemic model with the attitude evolution polarization model and regards the SIRS evolutionary process as follows: (1) from the individual $(S)$ affected by external factors, then the attitude values change $(I)$, to interact with the outside world to achieve a short balance $(R)$; (2) when the external information changes again $(S)$, it will intervene in the individual's attitude and view, urging the individual to continue to adjust the attitude $(I)$ and then maintain a relative balanced $(R)$ and recurring process with the outside world. Obviously, in the attitude evolution of each individual, the relative balance of attitude values is achieved in countless interactions with the outside world.

For the research of public opinion polarization, many scholars have made contributions to the study of group polarization behavior so far [19]. Their studies are mainly divided into two categories: (1) from the phenomenon itself, the qualitative analysis of the polarization behavior, the reasons for the polarization phenomenon, and the corresponding preventive measures are proposed at the macrolevel. (2) From the perspective of system dynamics, the quantitative analysis of polarization phenomena is studied. Some common models include D-W model [20, 21], J-A model $[22,23]$, and $q$-voter model $[24,25]$. The typical literatures are as follows. Flache et al. [26] proposed that a new generation of empirically based computational social influence models could make unique contributions for understanding key societal challenges, like the possible effects of social media on societal polarization. Li and Xiao [27] used the tunable BA network model closer to the real social network structure as the agent adjacency model and extended the individual's attitude value from one-dimensional to multidimensional based on the J-A classic model to establish multiple evaluation index systems. Bramson et al. [28] made quantitative analysis on the definition and measurement standard of polarization and put forward nine independent methods such as diffusion, dispersion, regionalization, and coverage to capture the polarization characteristics of distribution, providing a complete methodology for comparing the polarization degree across time and content. After that, they [29] further proposed that these nine phenomena might be regarded as polarization and then evaluated the polarization differences among the three types of models. Their research showed that the polarization degree in the cultural diffusion model of Axelrod family was positively correlated with the community disruption, while the polarization degree in the bounded confidence and relative consistency model was positively correlated with the community disruption and negatively correlated with the community disruption. In the structural equilibrium model of hierarchical trust transfer, the degree of polarization was related to community disruption, divergence, and group consensus, so there was no available calculation model to capture all nine polarization types. Wang et al. [30] used the limited trust model to develop a group-buying dynamic opinion model of the new technological product from group perspective in a multiagent complex system and explore the practical application of promoting the new technological product. Chen et al. [31] established the polarization model of public opinion considering the individual heterogeneity and dynamic integration and took the typical "Mimeng event" as an example to verify the rationality and effectiveness of their model. Sîrbu et al. [32] introduced a new model of opinion formation, which focused on the interplay between the possibility of explicit disagreement and modulated in a self-consistent way by the existing opinions' overlaps between the interacting individuals and the effect of external information on the system. They found that extreme information results in segregation and had a limited effect on the population, while milder messages have better success and a cohesion effect. Zhang et al. [33] integrated the influence factors such as conformity and network relationship strength into the polarization model, and the simulation results indicated that different information interaction modes had essential influence on group attitude polarization. However, in their study, the information propagation process of the polarization phenomenon was not considered. In addition, some scholars have studied polarization through social voting cases. For example, Stella et al. [34] analyzed large-scale social data collected during the Catalan referendum for independence on October 1, 2017, and identified the two polarized groups of independentists and constitutionalists and quantified the structural and emotional roles played by social bots. They found that bots act exacerbated social conflict online. In order to distinguish between users of opposing political alignments, Conover et al. [35] described several methods for predicting the political alignment of Twitter users based on the content and structure of their political communication in the run-up to the 2010 U.S. midterm elections. They introduced a support vector machine (SVM) trained on hash tag metadata and latent semantic analysis. Baumann et al. [36] proposed a model that introduced the dynamics of radicalization as a reinforcing mechanism driving the evolution to extreme opinions from moderate initial conditions and shed light on the mechanisms that might lie at the core of the emergence of echo chambers and polarization in social media. They found that the transition between a global consensus and emerging radicalized states was mostly governed by social influence and by the controversialness of the topic discussed. All these studies have better revealed the internal mechanism of polarization but more focused on the study of polarization, while ignoring the diffusion of information in the process of population polarization. Unlike these ones, this paper combines the current scenes of extreme publicity and high incidence in China and enhances the heterogeneity of individuals and the strength connection between heterogeneous individuals in multiagent simulation, in order to achieve the model closer to reality.

The above literature analysis shows that the current academic research on group polarization behavior has a certain depth, covering the fields of information science, 
computer science, and sociology. However, the abovementioned problems still exist. According to the research outcomes of the peer scholars, our study constructs the polarization model integrated with SIRS through combining the interaction among individuals and information dissemination in combination with the SIRS model. Also, as a statistical simulation method, the Monte Carlo method is suitable for the simulation experiment of discrete system and can be widely used to simulate the stochastic characteristics of the system $[37,38]$. Meanwhile, the group polarization process is a typical discrete process and we also take the network nodes (netizens) as agents. As a result, the multiagent Monte Carlo method is used to perform simulations under the social computing framework. In addition, the BA network is adopted to describe the interaction relations of netizens' attitudes due to its closer to the real social network structure. In doing so, this model describes the physical connection among individuals and integrates the information transmission in the polarization process, making the constructed model possibly closer to reality.

\section{Model Construction}

This section is focused on the modeling process of public opinion polarization in group behavior. Firstly, the classical polarization models named $\mathrm{D}-\mathrm{W}$ and J-A models are described, and their principles are also explained. Secondly, the SIRS epidemic model is introduced, and the roles in the SIRS epidemic model are transferred to the information diffusion scene. Finally, the extended polarization model combined with the SIRS epidemic one is proposed. Also, the relationship strength and other factors affecting public opinion polarization are also incorporated.

3.1. Classic D-W and J-A Models. The most classic models in the polarization model are the $\mathrm{D}-\mathrm{W}$ and the $\mathrm{J}$-A ones, while the J-A model better optimizes the D-W one, since it not only considers the assimilation effect in the social judgment theory but also considers the situations of repulsion and neutrality, Additionally, the J-A model is more appropriate with the polarization in reality and has a more scientific and practical meaning for the interpretation of the polarization process. It is specifically defined as follows:

(1) Assimilation rules:

If the attitude distance between the two randomly selected nodes $a$ and $b$ is within $d_{1}$, that is,

$$
\left|x_{a}-y_{b}\right|<d_{1} \text {, }
$$

then, the attitude values of the two nodes $a$ and $b$ change accordingly, and the updated formula is expressed as follows:

$$
\begin{aligned}
& X_{a}^{\prime}=x_{a}+\mu\left(x_{b}-x_{a}\right), \\
& X_{b}^{\prime}=x_{b}+\mu\left(x_{a}-x_{b}\right),
\end{aligned}
$$

where $\mu$ is the influence parameter $(\mu \in(0,0.5])$.
(2) Repulsion rules:

If the attitude distance between two randomly selected nodes $a$ and $b$ exceeds $d_{2}$, that is

$$
\left|x_{a}-y_{b}\right|>d_{2} \text {, }
$$

then, the attitude values of the two nodes $a$ and $b$ change accordingly, and the updated formula is as follows:

$$
\begin{aligned}
& X_{a}^{\prime}=\xi\left(X_{a}-\mu\left(X_{b}-X_{a}\right)\right), \\
& X_{b}^{\prime}=\xi\left(X_{b}-\mu\left(X_{a}-X_{b}\right)\right),
\end{aligned}
$$

where $\xi$ has a following definition:

$$
\xi(x)= \begin{cases}x, & 0 \leq x \leq 1, \\ 0, & x<0, \\ 1, & x>1 .\end{cases}
$$

(3) Neutral rules:

In other cases, the attitude values of the two nodes $a$ and $b$ do not change.

\subsection{SIRS Epidemic Model}

\subsubsection{Model Hypothesis}

(1) Combined with the significance of epidemic model and polarization model, this paper considers that the polarization of network and the diffusion of information are synchronous. In each information diffusion process, individuals will change their original attitude value because they accept the information transmitted by the surrounding individuals. Based on this, this paper uses the transformation of individual state in the infectious disease model to qualitatively determine the individual whose attitude changes after each information transmission. For example, if the individual state changes from $S$ to $I$, it means that the individual is infected and the attitude value will change accordingly. The polarization model further simulates the change of attitude value of infected individuals from the perspective of numerical dimension.

(2) Divide all individuals $(N)$ in the network into three categories, including individuals who are not affected by the environment of the public opinion, that is, healthy people $(S)$; individuals who are being infected with the public opinion and choose to change attitudes and disseminate information, that is, the infected people (I); individuals who are immune and stop spreading information to the outside after receiving the information several times, that is, the immunized people $(R)$.

(3) When a certain mass event occurs, the individual's attitude towards the event will be shaken by the 
initial uninformed $(S)$ to the influence of the surrounding infected individual, becoming a public opinion disseminator (I). The healthy one will become infected with the infection rate $\alpha$, and the infected individuals will change their attitude values, mainly including exclusion, assimilation, and neutral effect. However, the infected people will be immune after receiving the information several times, focusing on a new event or forgetting old one. They stop spreading information to the outside. Therefore, the infected people become the immunized people with the probability $\beta$. Also, some immunized individuals will be transformed into susceptible individuals $(S)$ with the probability $\gamma$ after being stimulated by new and powerful evidence information. It is possible for individuals to spread their ideas and make them susceptible again when new and powerful evidence information occurs. For example, Equifax Inc., one of the three largest consumer credit companies in the world, was involved in a scandal in 2017. In summer 2017, some news and media reported that part of consumers' credit information was breached by attacks. After this incident, people doubted the truth of the matter in a short time because Equifax was a reliable large size enterprise. They wondered whether the news was believable. Later, further report on news and media showed that cybercriminals accessed approximately 145.5 million U.S. Equifax consumers' personal data, including their full names, social security numbers, birth dates, addresses, and driver license numbers. People began to believe in the news and spread it $[39,40]$. We can see from this event, in the process of the development of the network mass event, the information disclosure will promote the roles of participants to constantly change.

(4) The proportions of the healthy people, infected people, and immunized people at time $t$ are $s(t), i(t)$, and $r(t)$, respectively. Obviously, $s(t)+i(t)+r(t)=1$; suppose $s(t), i(t)$, and $r(t)$ are continuous, differentiable functions for an event $T$ and the initial proportions of healthy, infected, and immunized ones are recorded as $s_{0}, i_{0}$, and $r_{0}$, respectively.

3.2.2. Establishment of the SIRS Model. According to the above hypothesis, the corresponding SIRS model is established, and the obtained differential equation is shown as follows:

$$
\left\{\begin{array}{l}
\frac{\mathrm{d} S(t)}{\mathrm{d} t}=\gamma n(R)-\alpha n(S), \\
\frac{\mathrm{d} I(t)}{\mathrm{d} t}=\alpha n(S)-\beta n(I), \\
\frac{\mathrm{d} R(t)}{\mathrm{d} t}=\beta n(I)-\gamma n(R), \\
N=n(S)+n(I)+n(R),
\end{array}\right.
$$

where $n(S), n(I)$, and $n(R)$ represent the number of healthy individuals, infected individuals, and immune individuals, respectively.

3.3. Extended Attitude Polarization Model. The J-A model considers the interaction of individuals and lacks the consideration of information transmission. Thus, this study incorporates the idea of the J-A model into the "susceptibleinfected" transmission stage of the SIRS epidemic model and reflects the information dissemination and diffusion process of public opinion polarization in group behavior based on the epidemic principle of infectious diseases. Subsequently, some measures are introduced to extend the original J-A model to make up its shortcomings. For example, different levels of relationship strength among nodes are used to describe the distinctions of intimacy relationships among real individuals. In addition, according to the characteristics of network public opinion, each node can obtain the attitude of the ones connected with it, so it is necessary to change the random pairwise interaction mode between individuals in the original model to the one-to-many interaction one among the connected individuals, so as to possibly reduce information asymmetry, making the individual choose one side with the support of sufficient information. Finally, we carry out experiments to simulate the evolution process of network nodes and get opinion emergence results by constructing the J-A model integrated into SIR model.

The variables and parameters involved in the model are defined as shown in Tables 1 and 2.

According to the above analysis, the specific process to build this model is demonstrated as shown in Figure 1.

(1) Use the SIRS model and formula (6) to simulate the information dissemination among individuals.

(2) Based on SIRS model, we clearly find the change of individual infection state. In this paper, SIRS model is used to determine the individuals whose attitude values have changed from state $S$ to $I$. We select the individuals whose statuses are healthy $(S)$ at time $t$, but at time $t+1$, the statuses are infected $(I)$, that is, the individuals converted from $S$ to $I$. In this model, these individuals have changed their attitudes during the information dissemination.

(3) The change from state $I$ to state $R$ and from state $R$ to state $S$ does not involve the further infection of the individual, and it will not produce the change of attitude value. Therefore, the most important part of the combination of polarization model and SIRS model is that individuals have health status and become infected. In this process, the infected individual is equivalent to receiving the information transmission from the surrounding individuals, and the attitude changes.

(4) After the individuals of attitude change are determined by SIRS epidemic model, this paper uses polarization model to calculate the variable of attitude quantitatively. Evolutionary rules of the individual's attitude value are expressed as follows. 
Table 1: Description of dynamic variables.

\begin{tabular}{lc}
\hline$X_{i}^{I}(t+1)$ & The attitude value of tde individual $i$ at time $t+1$ when it is in infection status $I$ \\
$X_{i}^{S}(t)$ & The attitude value of the individual $i$ at time $t$ when it is in health status $S$ \\
$X_{i}^{I}(t)$ & Individual comprehensive attitude value in infection status linked to individual $i$ at time $t$ \\
$X_{j}^{I}(t)$ & The attitude value of the individual $j$ at time $t$ when it is in infection status $I$ \\
$X_{i}^{I}(t)$ & Indicates the average attitude value of surrounding individuals \\
\hline
\end{tabular}

TABle 2: Parameter description.

\begin{tabular}{lc}
\hline$z_{i j}$ & Indicates tde network relationship strengtd \\
$\theta$ & Indicates assimilation parameter \\
$\vartheta$ & Indicates repulsion parameter \\
$d_{1}$ & Indicates assimilation boundary value \\
$d_{2}$ & Indicates repulsion boundary value \\
\hline
\end{tabular}

(1) Assimilation rules:

When the difference between the viewpoint held by the individual $i$ and the comprehensive viewpoint with the surrounding individuals is less than $d_{1}$, the attitude value of the individual $i$ is updated to

$$
\begin{aligned}
X_{i}^{I}(t+1)= & X_{i}^{S}(t)+\theta\left[X_{1}^{I}(t)-X_{i}^{s}(t)\right] \\
& \text { when }\left(\left|X_{i}^{I}(t)-X_{i}^{S}(t)\right|\right)<d_{1},
\end{aligned}
$$

where $X_{i}^{I}(t)$ is expressed as formula (8), $\theta$ represents the assimilation degree of attitude change, and the range is $(0,1)$ :

$$
X_{i}^{I}(t)=\frac{\sum_{j=1}^{n} z_{i j} X_{J}^{I}(t)}{\sum_{j=1}^{n} z_{i j}} .
$$

(2) Repulsion rules:

When the difference between the viewpoint held by the individual $i$ and the comprehensive viewpoint with the surrounding individuals is greater than $d_{2}$, the attitude value of the individual $i$ is updated to

$$
\begin{aligned}
X_{i}^{I}(t)= & X_{i}^{S}(t)-\vartheta\left[X_{1}^{I^{i}}(t)-X_{i}^{S}(t)\right], \\
& \text { when }\left(\left|X_{1}^{I}(t)-X_{i}^{s}(t)\right|\right)>d_{2} .
\end{aligned}
$$

(3) Neutral rules:

In other cases, the individual $i$ does not change its attitude value.

Moreover, the social judgment theory in the J-A model is also adopted as well as the assimilation and the repulsion effects. The above model is integrated into the SIRS epidemic model to better simulate the information transmission process of public opinion polarization in group behavior. At the same time, the model changes the pairwise interaction between individuals into one-to-many interaction, so that the information collection is relatively comprehensive.
The public opinion polarization model in group behavior proposed in this paper is shown in Figure 2, and the specific process is described as follows:

Step 1: according to different proportions, the individuals in the BA network are divided into healthy group $(S)$, infected group $(I)$, and immunized group $(R)$.

Step 2: a certain individual $X$ is selected randomly, based on the role $(S, I$, or $R$ ) of the surrounding individuals, the number of different roles, the infection rate $\alpha$, the immunization rate $\beta$, and the recovery rate $\gamma$ to comprehensively determine whether the individual $X$ changes his role and traverses all the individuals.

Step 3: according to the infectious disease model, we find out the individuals who have changed from healthy state to infected state and then determine the individuals who are infected. Then, when applied to the attitude polarization model, we confirm that these individuals have changed their opinions by the information transmitted by the surrounding individuals, that is, the individuals whose state has changed will change their attitude values because of the influence of the external information. As for the uninfected individuals, they are not interested in the information around them and are not willing to interact with each other.

Step 4: the infected individuals identified in Step 3 are substituted into the attitude polarization model to calculate the change of attitude value. Step 5: the above steps (2)-(4) are repeated for several times. Figure 3 presents the pseudocode of the simulation process and Figure 4 is the pseudocode of the SIRS model.

\section{Experimental Simulation}

In order to better reveal the process of mass event polarization intuitively, this study investigates different individuals' evolutionary attitude process in the group through simulation experiments. Firstly, according to the above model, the extended BA network which introduces acquaintance recommendation mechanism is selected to simulate public opinion polarization. The reason is that the nodes of the social network follow the power-law distribution and they have the feature that a very small number of individuals with a large audience and higher clustering coefficient. Therefore, the extended BA network is used to 


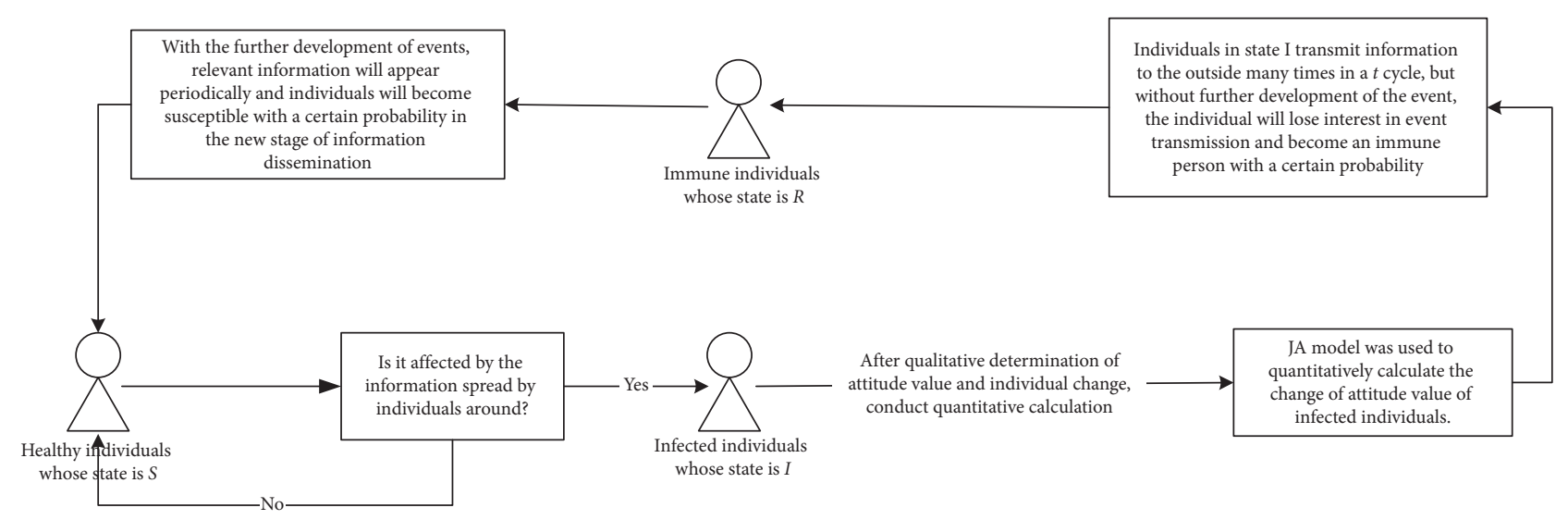

FIgURE 1: The principle of the polarization model integrated with SIRS.

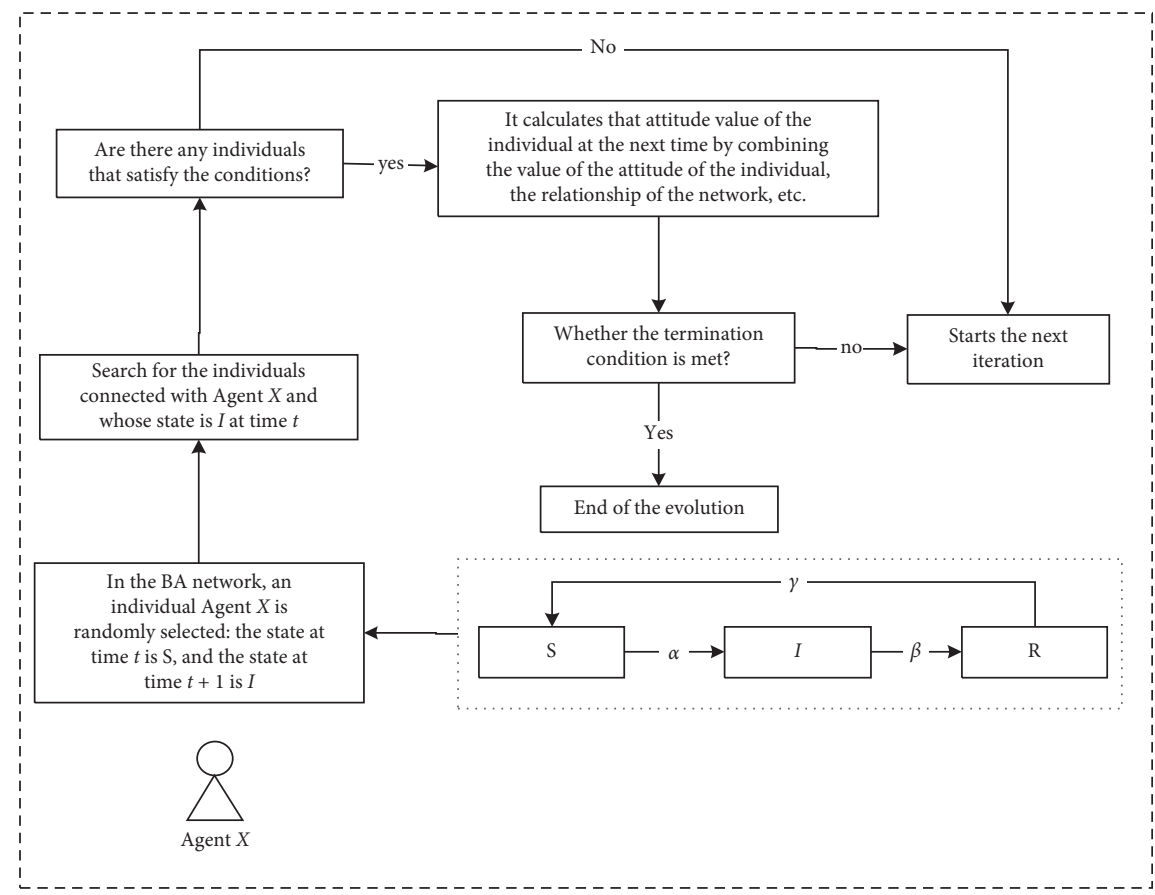

Figure 2: Public opinion polarization model in group behavior.

reflect the real social network. The network structure and corresponding data are shown in Figure 5 and Table 3. Through several simulation experiments, we find that the 300 -node simulation network effectively simulates the information diffusion and polarization process, and the experimental process is more efficient. Therefore, the size of the network is set to $300 . d_{1}$ and $d_{2}$ represent the boundary values of assimilation and exclusion, respectively. In order to simulate the polarization process of group events with significant impact, we set smaller assimilation domain $\left(d_{1}=0.3\right)$ and larger exclusion domain $\left(d_{2}=1.1\right)$ to reflect the intensified contradiction. After 400 interactions, the attitude changes along the surrounding environment, some of individuals still maintain the original attitudes, while some are polarized, and the rest adjust their attitudes to strike a balance with the surrounding environment. The evolution results of the infected individual proportion and individual attitude values in the SIRS model with the interaction time is shown in Figures 6 and 7:

The average shortest path length $l_{G}$ is

$$
l_{G}=\frac{1}{n(n-1)} \cdot \sum_{i, j} d\left(v_{i}, v_{j}\right),
$$

where $G$ represents the BA network and $n$ is the number of vertices in $G$.

The clustering coefficient $C_{G}$ is

$$
C_{G}=2 * \frac{1}{n} * \sum_{i=1}^{n}\left(k_{i} *\left(k_{i}-1\right)\right) \text {. }
$$

The mean of degree $D_{G}$ is

$$
D_{G}=\frac{1}{n} \sum_{i=1}^{n} k_{i}
$$




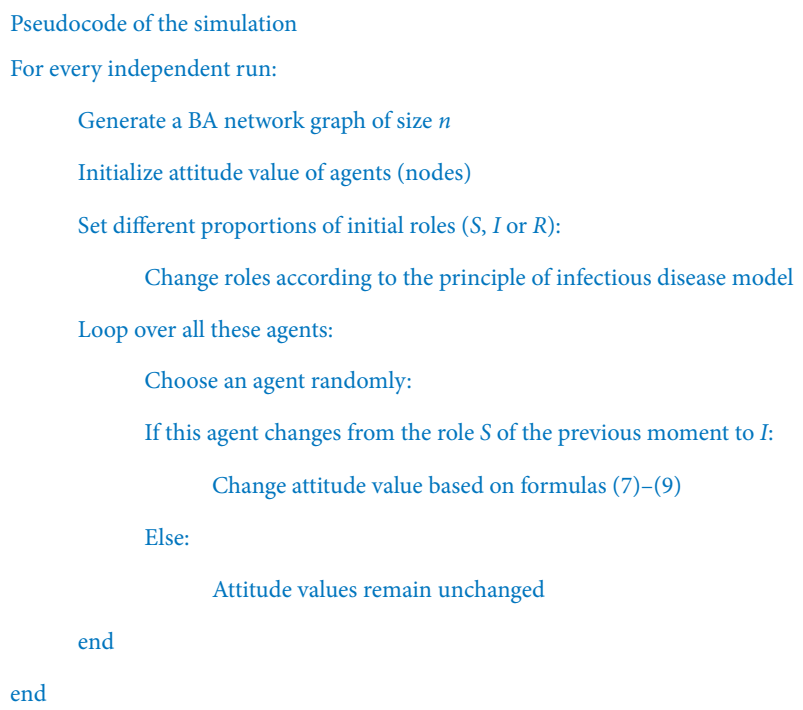

Figure 3: Pseudocode of the simulation process.

where $k_{i}$ is the degree of $i$ in $G$.

In Figure 6, the horizontal coordinates standing for the number of interactions and the vertical coordinates represent the proportion of individuals participating in the group polarization and their changing process for the attitudes during the information interaction process. It is not difficult to find that the infected individuals fluctuate between 0 and 0.5. As shown in Figure 7, the horizontal coordinates and vertical coordinates represent the number of interactions and individual attitude values, respectively. From the simulation results, the individual gradually develops a polarization trend after intense interactions, and the attitudes begin to shift to the two extreme directions 1 and -1 , while a small number of individuals adhere to their own opinions without being affected by the surrounding environment. The points representing their attitude values are scattered between -1 and 1 . However, since the overlapped parts of the evolutionary process of individual attitude values cannot be visually reflected in the graph, we also give the individual attitude distribution under different interaction times (notice that time is used to measure the number of interactions), as shown in Figure 8.

The histogram for individual distribution of the initial state time $=0$ is shown in Figure 8(a), in which the horizontal coordinates indicate the individual's attitude value and the vertical coordinates represent the number of individuals. In the initial stage, the distribution of individuals' attitude values is relatively uniform, and the understanding and viewpoint of the event are relatively scattered. However, when the depth of interindividual communication is greater, the evolutionary process of time from 0 to 400 illustrates that the whole group gradually presents two levels of differentiation. The opinion polarization phenomenon gradually emerges, but there are also very few individuals who hold different opinions. The main reasons are as follows: (1) the contradictions exposed by mass events are sharper and the impact is larger, which make the individual easy to repel and the assimilation is weak, causing two extremely dominated opinions. (2) A few individuals have strong self sense. The influences exerted by the outside world are weak for them, and they always maintain their own inherent attitudes. (3) Some individuals have strong immunity to external interference. They rarely receive outside information, and thus have low participation in the discussion of mass events.

\section{Results and Discussion}

This section mainly discusses the following aspects based on the experimental simulation results: (1) it analyzes the influence of SIRS-based information diffusion on the polarization process of group attitudes and verifies the significance of polarization effects with and without integration of the SIRS epidemic model; (2) it explores the relationship between group polarization and individual immunity to information by adjusting the recovery rate parameter $\gamma$ in the SIRS epidemic model; (3) it explores the influence of different relationship strength $Z$ on polarization effect; (4) it investigates the effects of different network structures on polarization. Exploring the above four aspects further analyzes the important factors affecting the development and diffusion of public opinion polarization in group behavior, which will be used for reference to reduce the social harm of extreme network mass events.

5.1. Comparing the Polarization Process with and without Integration of SIRS Epidemic Model. This section explores the impact of the transmission characteristics of epidemic on the group polarization effect, which is achieved by controlling network structure, initial attitude values, and other parameters to remain unchanged, while only changing the way of information interaction among individuals. The model uses epidemic transmission to carry out individual information interaction, as shown in Figure 9(a), and the epidemic model is also used to screen out nodes in different state stages. We define that the individual whose state is changed from state $S$ to $I$ at the next moment is affected by the surrounding individuals. If they change from susceptible individuals to infected ones, it is obvious that their attitudes have been changed. Subsequently, by searching their connected individuals with states $I$ at last moment and then integrating the attitude values of these individuals, the amount of change is calculated. The information interaction mode of the comparison model is shown in Figure 9(b). The intermediate individual ' $A$ ' needs to exchange information with all the surrounding individuals before judging whether to change his altitude value. Also, the individual ' $A$ ' synthesizes the opinions of all the surrounding individuals and updates his attitude value. Comparing the polarizations with the epidemic mode to the one with one-to-many mode, the effect is shown in Figures 10 and 11.

Comparing the polarization effects of the two modes, it can be found from Figures 10 and 11 and Table 4 that these two ones both have relatively obvious polarization trends, and the polarization effects are significant. However, comparing Figures 10(a) and 10(b), it can be found that the concentration of opinions of epidemic mode is higher than 


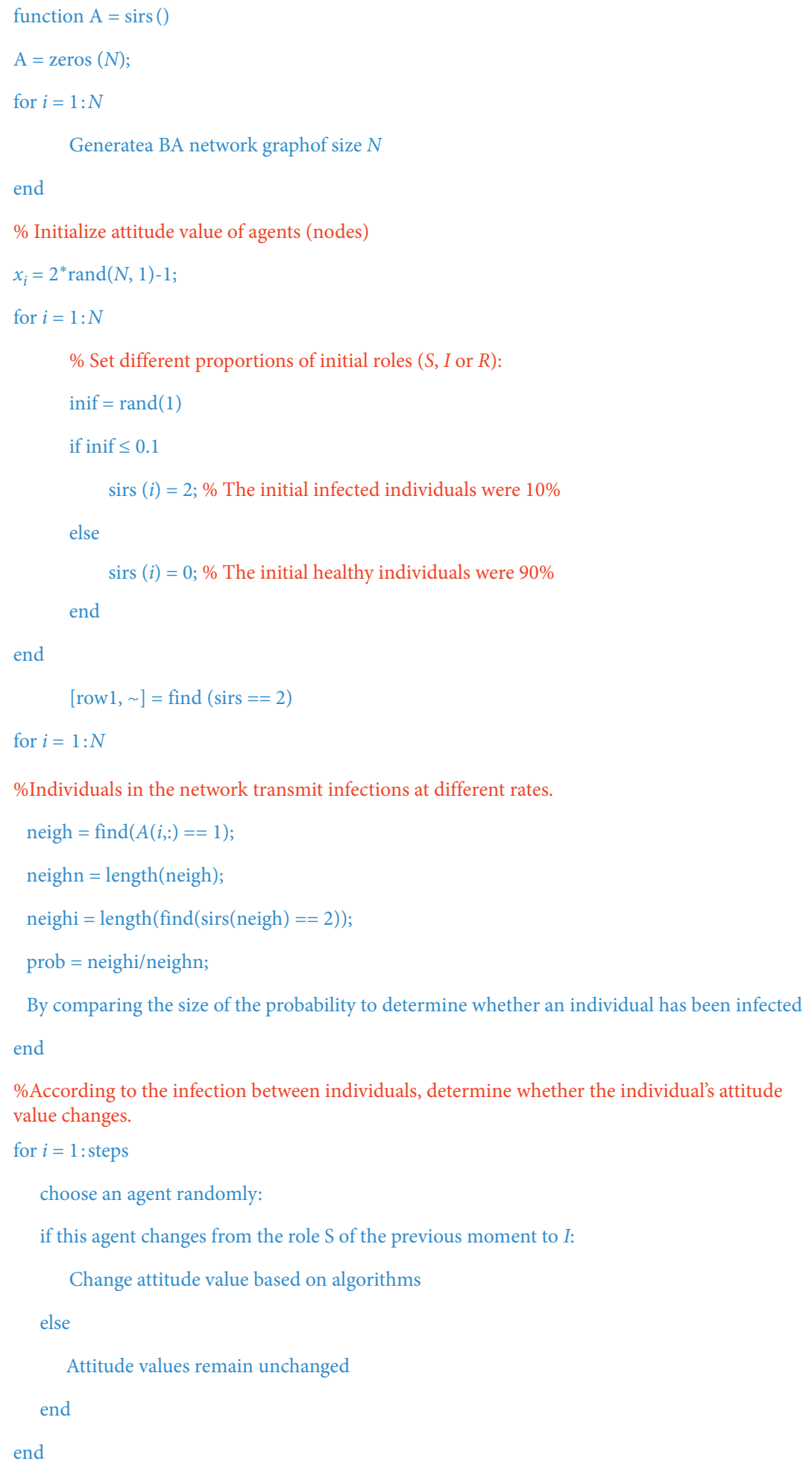

FIgure 4: Pseudocode of the SIRS model.

that of one-to-many mode, and the polarization speed in epidemic mode is faster. Thus, the model with integration of the SIRS epidemic mode has a better polarization effect under the same interactions. The reason lies in that different modes of information interaction among individuals can greatly influence the final polarization effect. In connection with the reality of life, when individuals face a topic of public opinion, they should listen to the opinions of all the individuals around them as many as possible, which will lead to the neutralized idea tend to be rational, and the extreme trend will slow down unless the surrounding individuals have formed a more obvious polarization perspective from the beginning. But the possibility of this situation is low, so the final polarization process is slower too; while the 


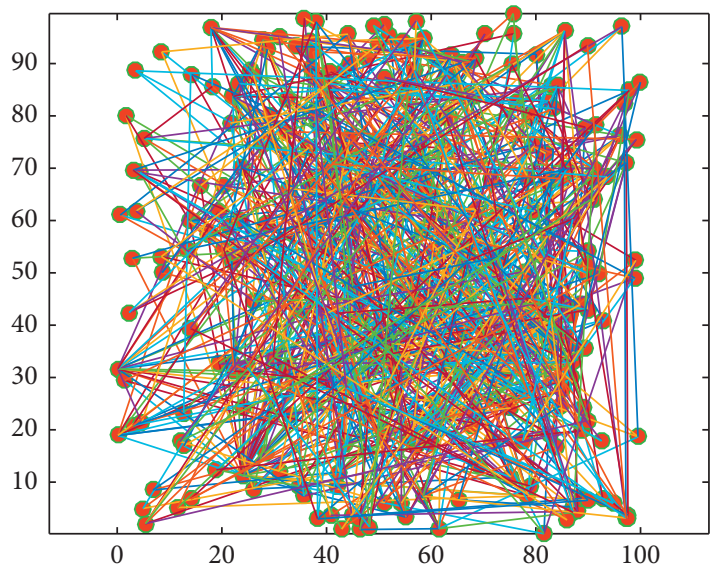

(a)

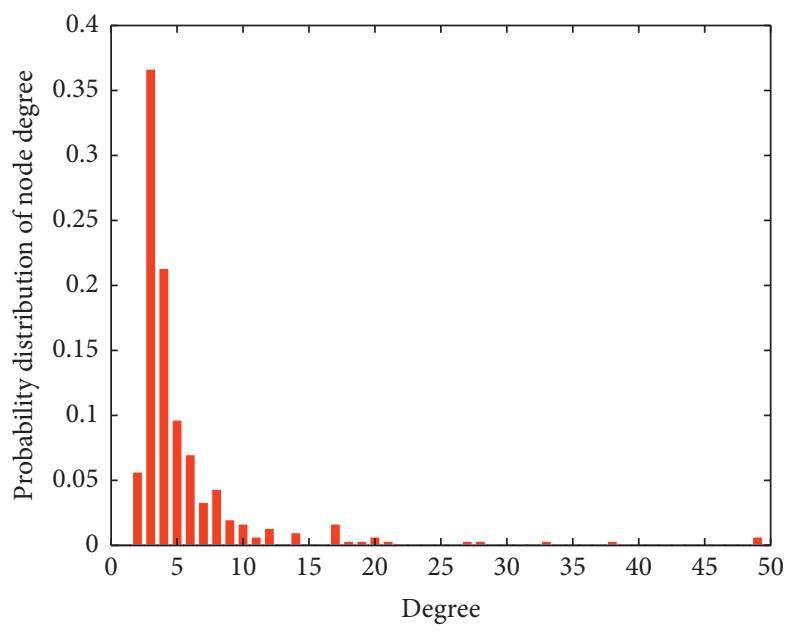

(b)

Figure 5: Schematic diagram of network structure. (a) Extended BA network structure diagram. (b) Probability distribution of node degree in network.

TABLE 3: Parameter statistics of network structure corresponding to Figure 5.

\begin{tabular}{lcc}
\hline $\begin{array}{l}\text { Average shortest } \\
\text { path length }\end{array}$ & Clustering coefficient & Mean of degree \\
\hline 3.6978 & 0.61217 & 5.6267 \\
\hline
\end{tabular}

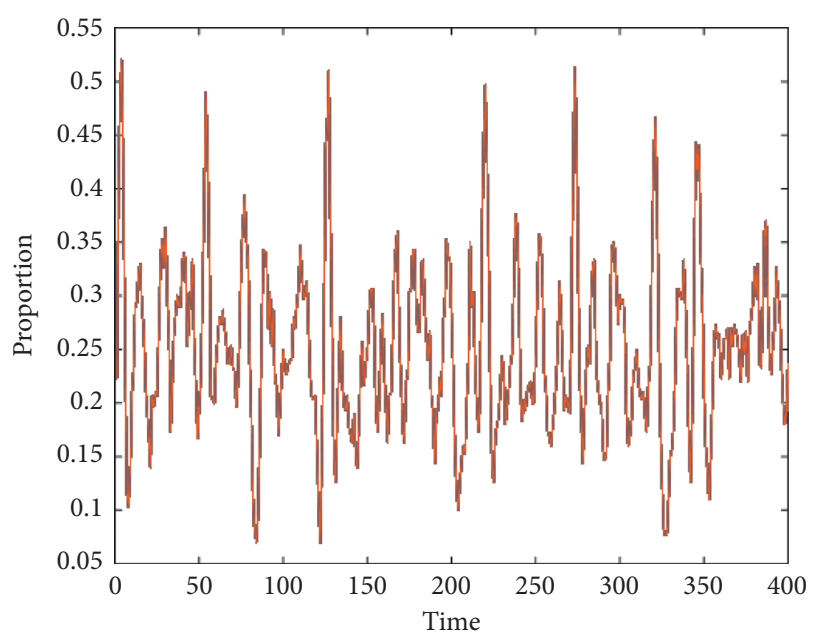

FIGURE 6: Infected individual proportion.

individuals who change from $S$ to $I$ state are screened through the epidemic model, they consider the change of individual attitude as the process of infection. Through the information dissemination, the communicator infects the surrounding noncommunicators with a certain probability, which in turn affects the opinions of the noncommunicators and enables them to become communicators who continue to spread the information. This format makes the change of individual attitude only be affected by the surrounding communicators, which is easy to form the strong and weak opinions. Generally, the individual's conformity leads to compromise and yield to the strong side, and ultimately the

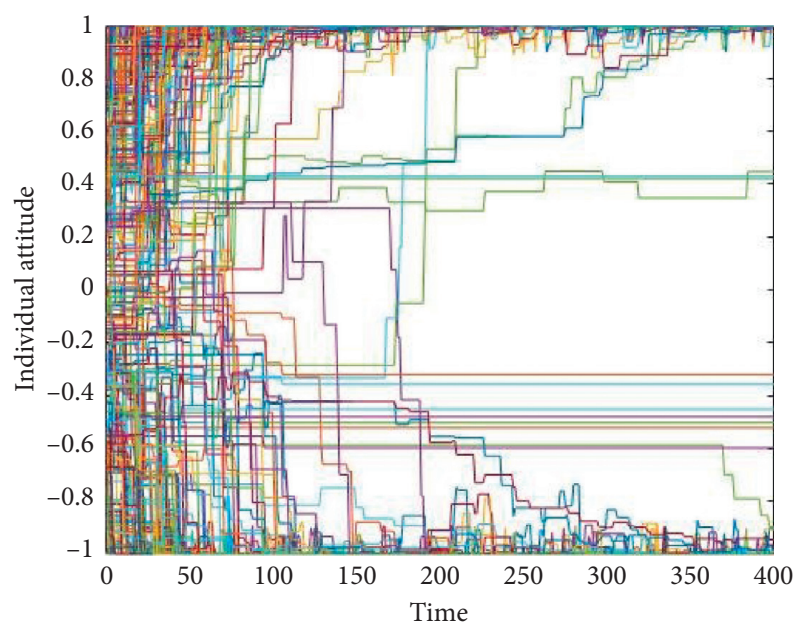

FIgURE 7: Individual attitude with number of interactions.

decisions made by them tend to follow the strong side, thus the polarization emerges.

In addition, since infectious viruses are difficult to control in the process of transmission, the spread of public opinion with epidemic characteristic exerts a large influence on the society. Usually, it attacks all nodes in the network during the transmission process and spreads rapidly to the entire network, making the individuals vulnerable to the extreme public opinion.

5.2. Effect of Immune Recovery Rate $\gamma$ on Polarization Effect. In reality, with the official refutation of false information and the improvement of individual cognitive level, the individuals' willingness to spread information is constantly reduced, and then the information dissemination is stopped to reach the immune state. However, there are some mass events with great influence. Due to the opacity of official channel information in the early stage of the event, the 


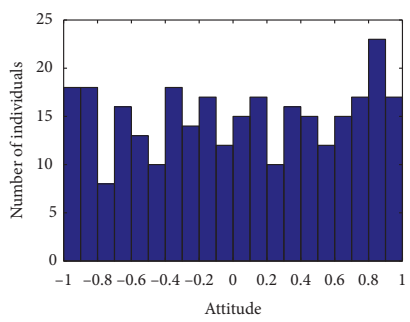

(a)

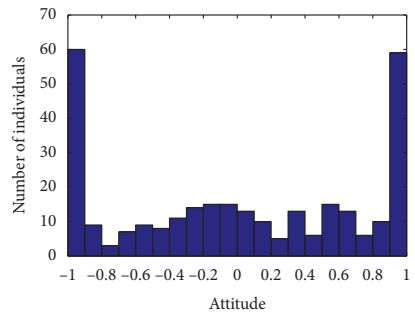

(b)

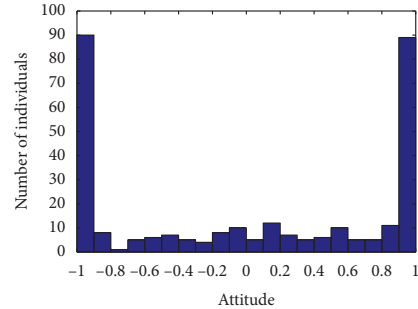

(c)

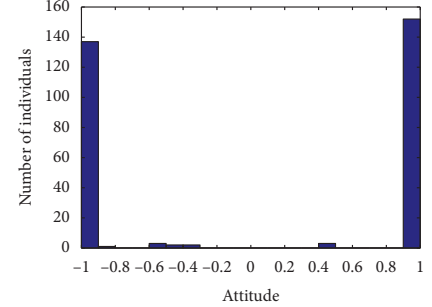

(d)

Figure 8: Individual attitudes under different interaction times. (a) Time $=0$. (b) Time $=50$. (c) Time $=100$. (d) Time $=400$.

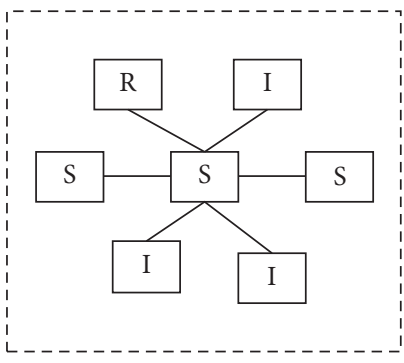

(a)

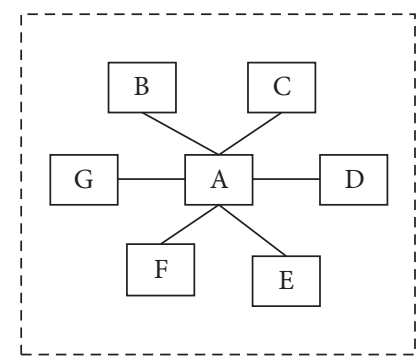

(b)

FIGURE 9: Individual information interaction mode in polarization model. (a) Information interaction Mode (epidemic model). (b) Information interaction mode (one-to-many mode).

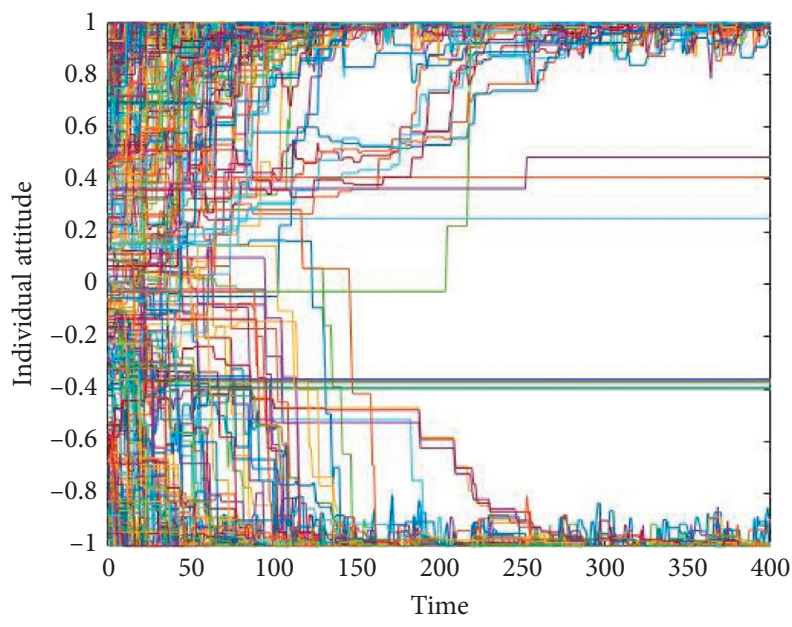

(a)

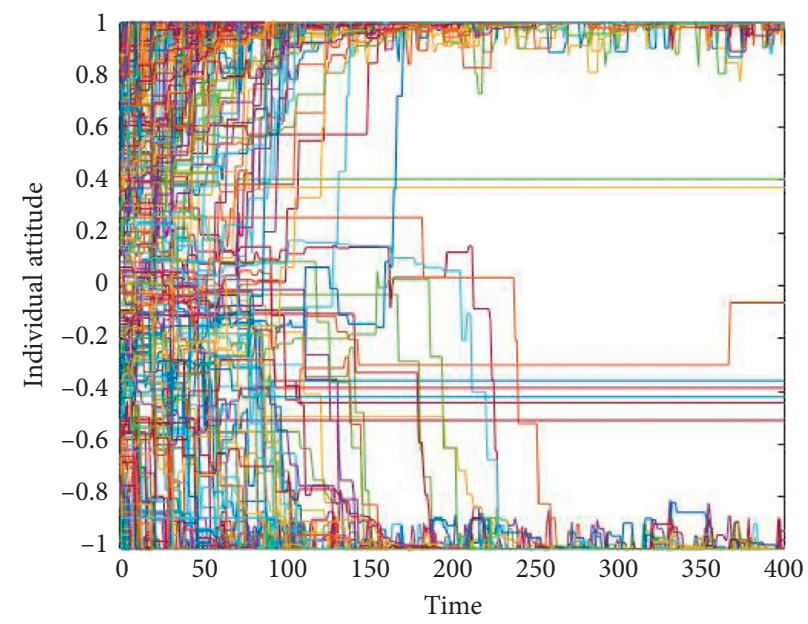

(b)

FIGURE 10: Individual attitudes with interactions in different interaction modes. (a) With epidemic mode. (b) With one-to-many mode.

public has doubts about the credibility of the government, which makes the participating groups unable to obtain the authoritative information to support the event. As a result, different versions of information are constantly emerging, and individuals are forced to adjust their attitudes and standpoints according to the relatively reliable information sources, forming a phased closed-loop similar to SIRS infectious diseases. The immunity of individuals to information, to a large extent, depends on the amount of information disclosed and the degree of authority. Based on this, this section explores the effect of the recovery probability from an immunized individual to a susceptible individual on the final polarization and controls the immunity rate of immunized individuals by increasing and decreasing the $\gamma$ value. In this case, other conditions remain unchanged. The parameter $\gamma$ value is adjusted, the recovery rate is increased from 0.1 to 0.5 , the step size is set to 0.2 , and the health, infection, immunized individual ratio curve, and polarization effect diagrams are observed, respectively. 

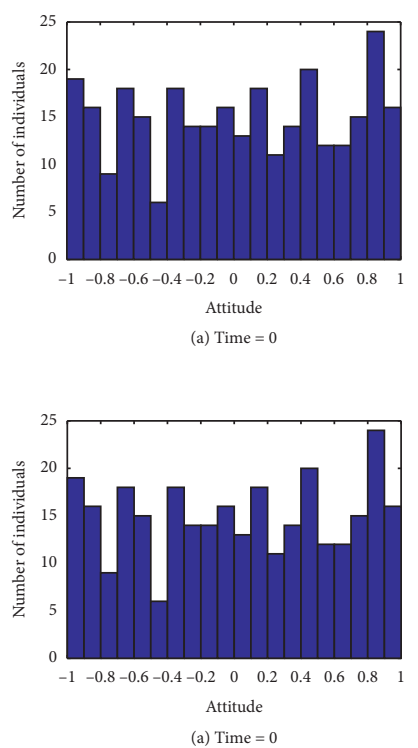
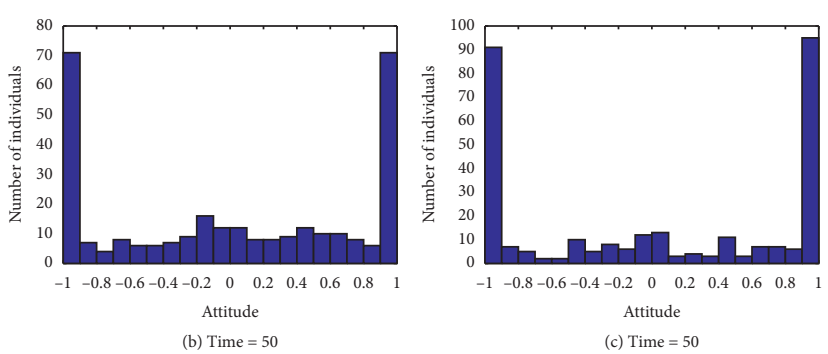

(a)

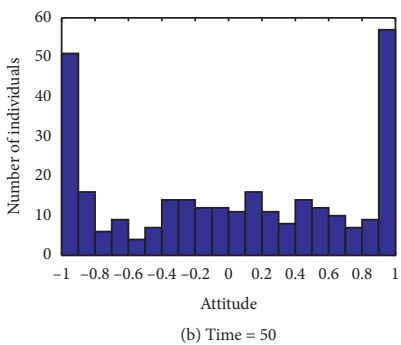

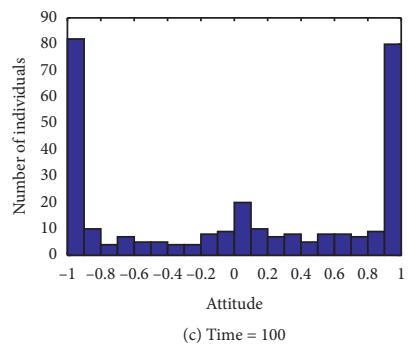

(c) Time $=100$
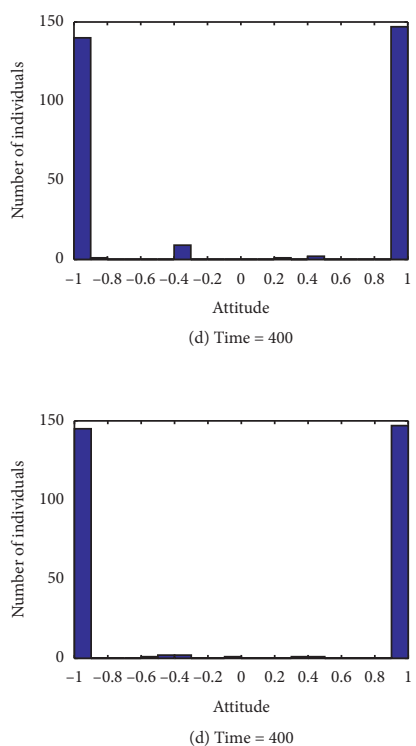

(b)

Figure 11: Individual attitude distribution under different interaction times of polarization model. (a) Epidemic interaction model. (b) Oneto-many mode.

TABLE 4: Statistical table of individual polarizability corresponding to the two modes in different interaction stages.

\begin{tabular}{lcccc}
\hline Time & 0 & 50 & 100 & 400 \\
\hline Epidemic interaction model & 16.1 & 48.3 & 63 & 97.7 \\
One-to-many mode & 16.1 & 36.7 & 54.7 & 94 \\
\hline
\end{tabular}

As shown in Figure 12, when the immune recovery rate are 0.1 and 0.3 , respectively, the degree of information dissemination gradually increases, while the individuals tend to be stable after a certain interactions, and the information disseminators in the group disappear, leaving only healthy individuals. When the immune recovery rate is increased to 0.5 , the three roles in the individuals are constantly reciprocating, and the spread of information is similar to the strong infectious virus, which has always existed in the group. We can also see from Figures 13 and 14 that as the recovery rate increases, the polarization effect tends to increase. Therefore, it is easy to find that the increase of the immune recovery rate $\gamma$ makes the information transmission from the beginning to the steady state lasts longer, and the final polarization effect and the polarization speed are obviously improved. The internal mechanism of the group polarization process is also analyzed by means of the simulation results. On one hand, from the perspective of epidemic transmission, when individuals have high immunities to an epidemic, the spread of the epidemic will be limited and the infection rate as well as the infection scope will also be controlled effectively. On the other hand, from the perspective of information transmission, when the individuals have high immunities to the public opinion, the influence of public opinion on individuals will be greatly reduced, and the spread of news will be greatly weakened as well. Therefore, the spread of public opinion will be curbed. Similarly, the significance of the viewpoints of group polarization in the model is affected by the breadth and depth of information transmission among individuals. The group polarization effect will be less significant if the information transmission is blocked.

According to the analysis mentioned above, in the prevention or resolution of mass events, the strategies that may be included as follows: (1) to improve the individual's immunity to public opinion information; (2) to effectively release the authoritative information from various channels; (3) to enhance the individual's immunity to false statements; (4) to accurately identify the authenticity of the information. Consequently, the individual will be prevented from becoming a communicator of false news.

\subsection{Influence of Relationship Strength Z on Polarization Effect.} This section is focused on the impact of the relationship strengths among individuals on the polarization process. Since the relationship strength is used to describe the close relationship among nodes, that is, the close relationship among people in the real society, it is not only reflected as the weights in a graph but also involves the gaming of interpersonal relationship of psychology. The closer the relationship is, the greater the impact on each other is. According to this, the relationship strength, as an important part of the complex network, has a greater impact to the development of polarization.

Because the nodes in the BA network follow the power-law distribution, which has the characteristics of small audience and high clustering coefficient, it is used to simulate the real social network. This section mainly keeps other variables unchanged and compares the influence of different relationship strengths on the polarization results in BA network and thus derives its influence on the polarization process. Considering the scientific nature of the experiment, this paper has carried 


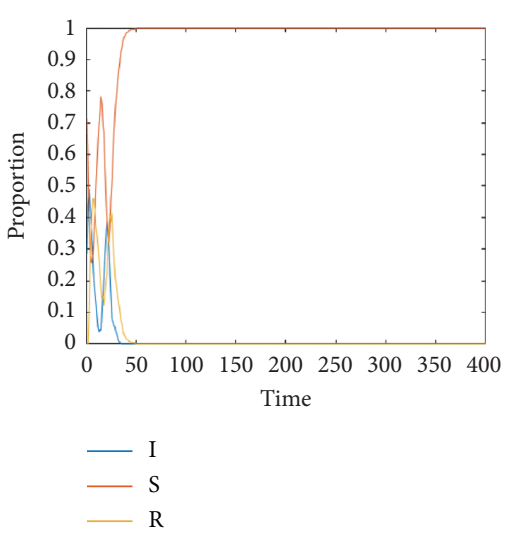

(a)

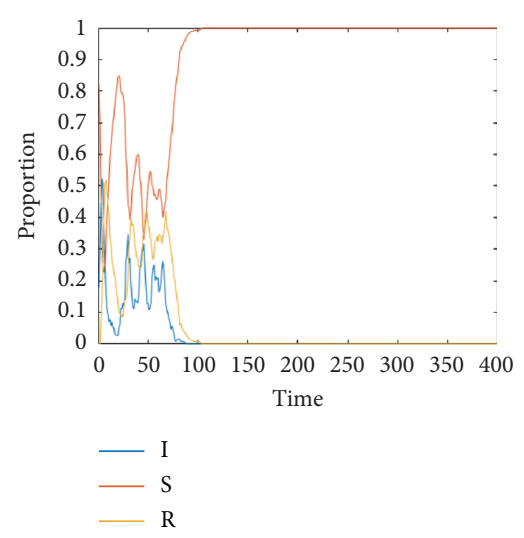

(b)

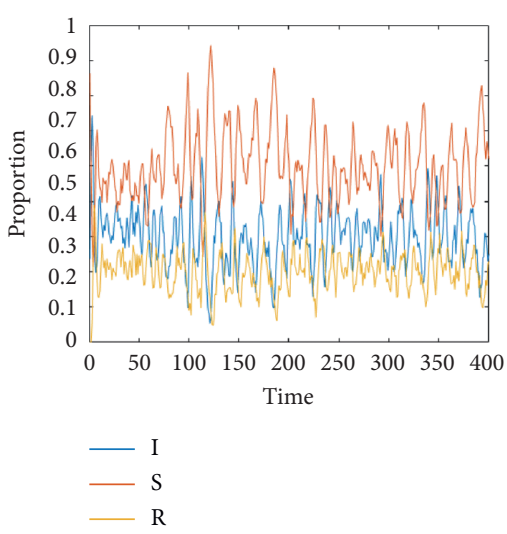

(c)

Figure 12: Transmission curve of epidemic model with immune recovery rate of $0.1,0.3$, and 0.5 . (a) $\gamma=0.1$. (b) $\gamma=0.3$. (c) $\gamma=0.5$.

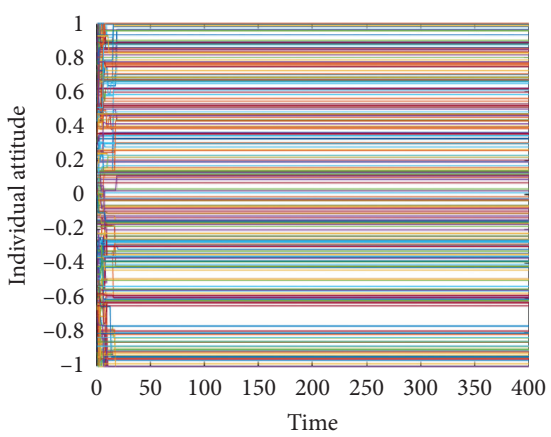

(a)

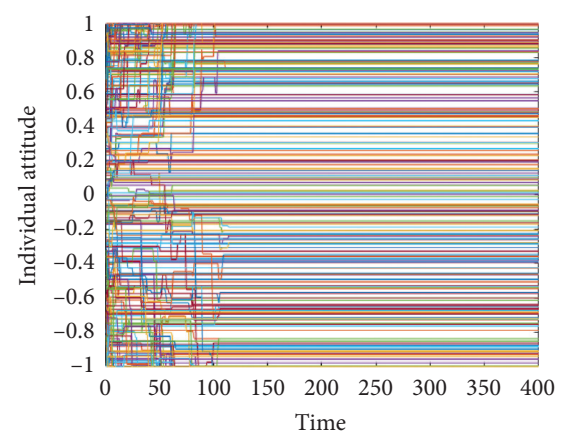

(b)

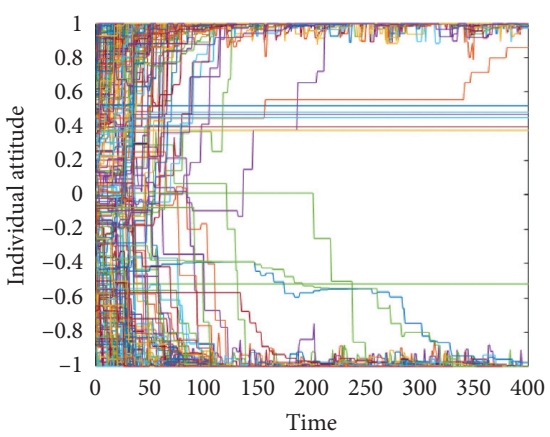

(c)

FIGURE 13: Attitude polarization evolution with immune recovery rate of $0.1,0.3$, and 0.5. (a) $\gamma=0.1$. (b) $\gamma=0.3$. (c) $\gamma=0.5$.

out several times of repeated simulation experiments and designed four groups of experimental objects. One chooses homogeneous strength with the relationships between connected individuals as 2 , and others choose heterogeneous strength with the relationships to a random number between $1-4,1-6$, and 1-8. All the other variables remain unchanged. The simulation was repeated several times, and the obtained average results are shown in Figure 15.

As shown in Figure 15 and Table 5, on the whole, the polarization proportion of heterogeneous relational strength network is similar to that of homogeneous relational strength network. However, in the early stage of interaction, that is, before the number of interactions is less than 150 times, the speed of group polarization in relation strength heterogeneous network is relatively fast. When the attitude polarization of groups tends to be stable, the influence of homogeneity and heterogeneity of relation strength on polarization is relatively small. Just like a topic discussion in a meeting, all individuals have their own opinions initially. If this process is regarded as a polarization process, when the participants are strangers to each other, it will be relatively difficult to unify the points or eventually divide them into several relatively consistent points of view, because each individual, as a decisionmaker, needs to integrate the individuals with similar views equally. If the participants are familiar with each other and there are common interests or close relationships among individuals, then the decision-making individuals can choose the decision-making of individuals with similar or closely related views by weight. In the process, the assimilation and exclusion of views will be more obvious, and the final decision-making results will appear faster.

Therefore, when dealing with the polarization of public opinion, it is necessary to analyze the composition of the group, so as to understand the interests and emotions among individuals in the whole group as the judgment indicators of the relationship strength. If the relationship strength among individuals is larger, the polarization of the event will be relatively faster.

\subsection{Influence of Different Network Structures on Polarization} Process. During the process of investigating public opinion polarization in group behavior, network structure as an important factor is of great significance to its evolutionary result. Different network structures represent different ways of information exchange and transmission among groups, which have a greater impact on the information transmission, interaction, and polarization of opinions. As a result, in this section, we select three different networks including BA, 

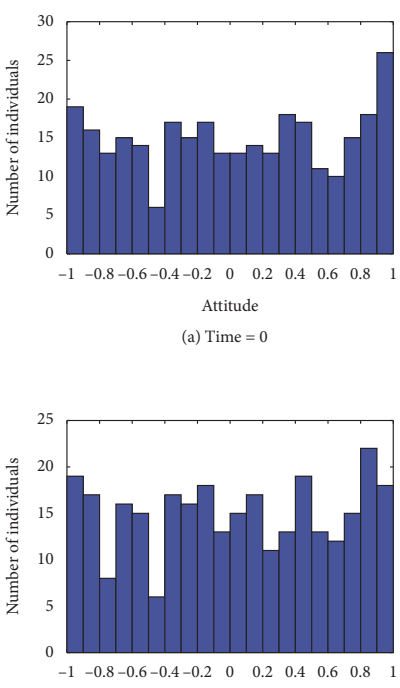

Attitude

(a) Time $=0$

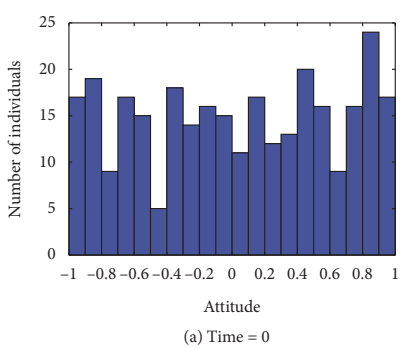

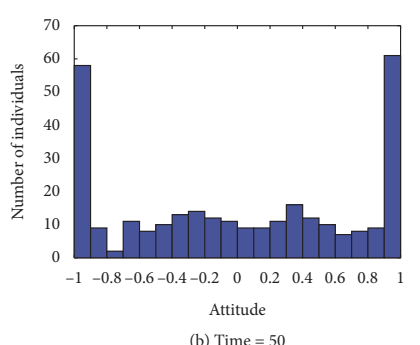

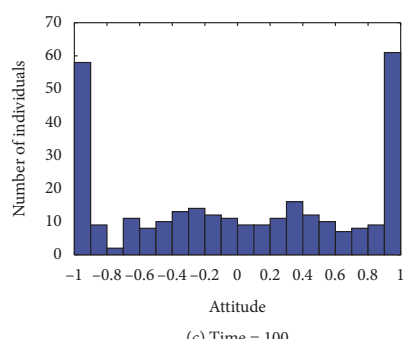

(a)

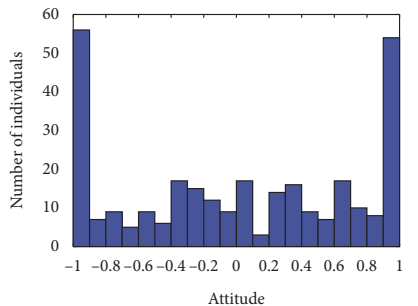

(b) Time $=50$

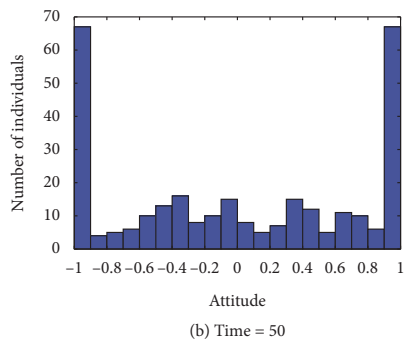

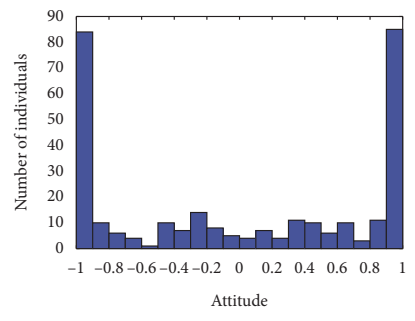

(c) Time $=100$

(b)

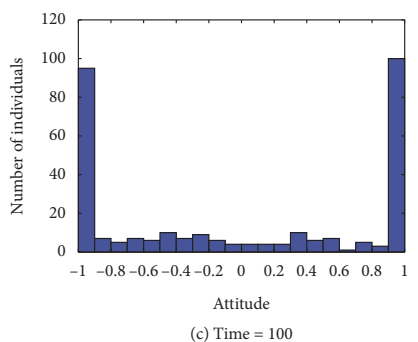

(c)
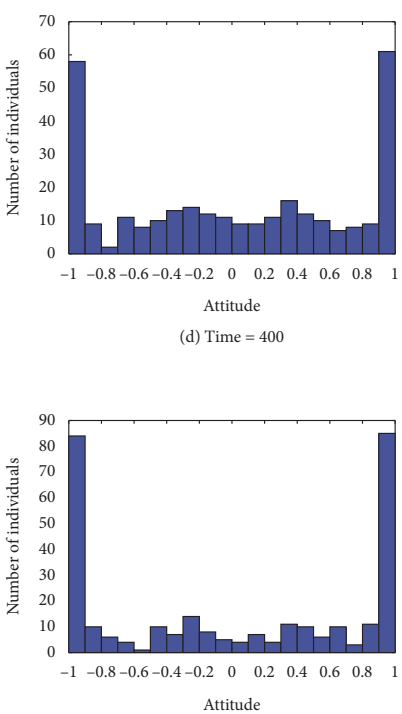

(d) Time $=400$

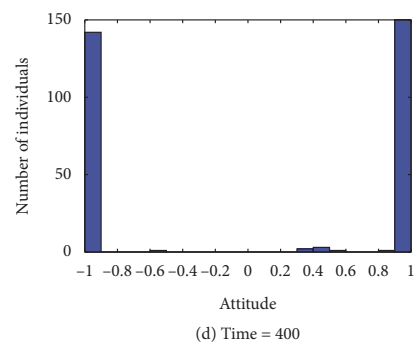

FIGURE 14: Histogram of individual attitude distribution under different interaction times corresponding to Figures 13 (a)-13(c). (a) $\gamma=0.1$. (b) $\gamma=0.3$. (c) $\gamma=0.5$.

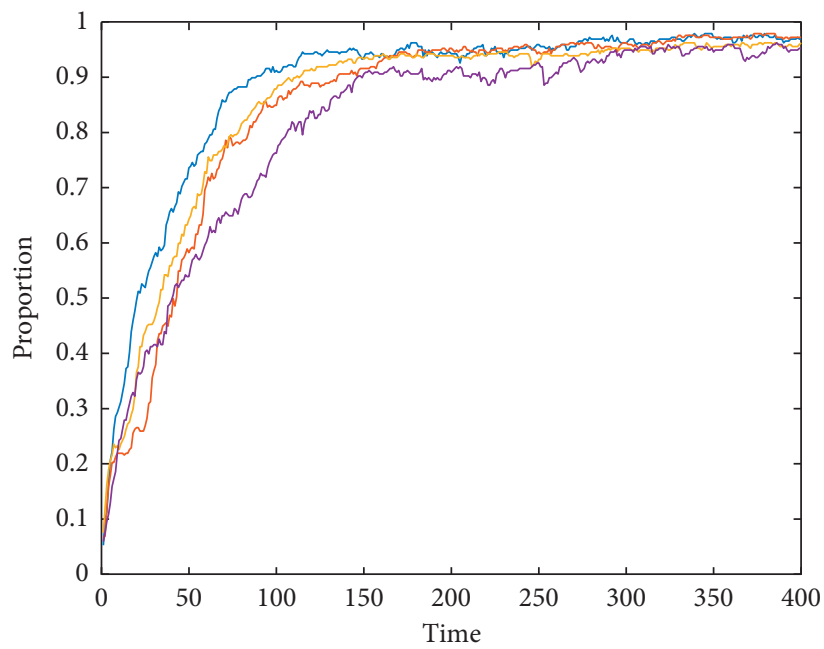

- Strength of heterogeneity relationship obeying $1-4$

- Strength of heterogeneity relationship obeying 1-6

Strength of heterogeneity relationship obeying 1-8

- Homogeneous relationship strength

Figure 15: Comparison of attitude polarization between networks of homogeneous and heterogeneous relationship strengths. 
TABle 5: Statistical table of polarization ratio of individuals under different relationship intensities (mean value of 5 experiments).

\begin{tabular}{|c|c|c|c|c|c|c|}
\hline Time & $0(\%)$ & $50(\%)$ & $100(\%)$ & $150(\%)$ & $200(\%)$ & $400(\%)$ \\
\hline Homogeneous relationship strength & 6.3 & 52.7 & 75.4 & 88.0 & 89.8 & 95.4 \\
\hline Strength of homogeneity relationship obeying 1-4 & 6.3 & 75.2 & 90.6 & 92.1 & 92.2 & 96.7 \\
\hline Strength of homogeneity relationship obeying 1-6 & 6.3 & 58.9 .7 & 83.7 & 89.1 & 92.4 & 96.7 \\
\hline Strength of homogeneity relationship obeying $1-8$ & 6.3 & 64.1 & 85.3 & 91.7 & 92.2 & 96.1 \\
\hline
\end{tabular}

small world (SW), and fully connected networks (FCN) to study the influence on opinion polarization.

We firstly construct the fully connected network and the small-world network. The fully connected network is shown in Figure 16. The small-world network is built as follows: the nearest neighbor coupling networks are generated, including $N$ nodes and connecting multiedges, in which each node is connected with $K / 2$ nodes of its left and right neighbors, where $K$ is an even number. Later, taken the random number $p(p \in(0,1))$ as the probability, each node reconnects every single edge of the initial network. But in the rewiring process, it is forbidden to have multiple connections between two nodes or exist the self-connecting of the same node shown in Figure 17.

Comparing the public opinion polarization process in group behavior under the BA network, the small-world network, and fully connected network, the impacts of different network structures on the degrees of group polarization are visually shown in Figures 18-20. Considering the reliability of the experiment, the size of the BA network, small-world network, and fully connected network should be consistent meaning these different networks have the same number of nodes as well as edges. Meanwhile, several experiments should be carried out to compare the polarization processes under the three networks, and the results are shown in Figures 18-20.

In Figures 18(a), 19(a), and 20(a), the polarization processes of BA network, small-world network, and fully connected network are compared. When we adjust different network connection parameters, the experimental results show that the polarization effect under BA network is obviously better than that of small-world and fully connected network. Also, the polarization degree of fully connected network is the lowest. In Figure 18(a), if the probability of edge-breaking reconnection parameter $p$ of the small-world network is set to 0.1 , the difference between the polarization effect under the BA network is small as a whole; and when $p$ is adjusted to 0.05 and 0.01 (As shown in Figures 19(a) and $20(\mathrm{a})$ ), the polarization effect is significantly reduced. In addition, Figures 18(b), 18(c), 19(b), 19(c), 20(b), and 20(c) represent the polarization trajectories of the participants in BA and SW networks, respectively.

In this paper, ten independent simulation experiments are carried out under different network structures to observe the variance of attitude distribution of network individuals in each timeslot. In order to make the experiments more accurately and ensure the rationality, the maximum and minimum values in the calculation results are removed, and then the average value is taken for calculation. Table 6 is the results for comparing the average difference of group

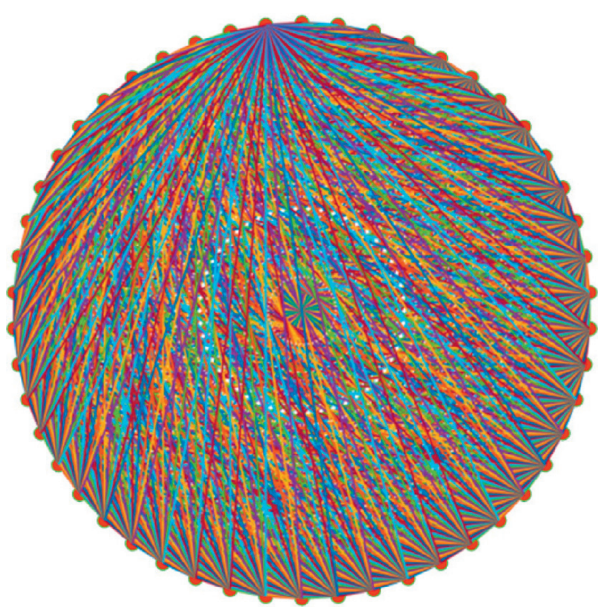

FIgURE 16: Fully connected network.

attitudes in different timeslots under different network structures. The calculation formula is as follows:

$$
\begin{aligned}
M & =\sum_{i=1}^{n} \frac{x_{i}}{n} \\
S_{1}^{2} & =\frac{\sum_{i=1}^{n}\left(M-x_{i}\right)^{2}}{n}, \\
D & =\frac{\sum_{j=1}^{10} S_{j}^{2}-\min \left(S_{j}^{2}\right)-\max \left(S_{j}^{2}\right)}{8},
\end{aligned}
$$

where $M$ is the mean of attitudes, $S_{1}^{2}$ is the variance of attitudes, and $D$ is the mean of variance after removing the maximum and minimum values.

The statistical results show that with the development of polarization process, the average difference of views and attitudes of groups is continuously enlarged. The reasons are as follows: (1) the structure of BA network determines the speed of information transmission and the vulnerability to risk when dealing with the invasion of news of a similar epidemic. If the degree of some nodes is extremely large, once these ones are invaded, the speed for which the network is infected by information will be greatly accelerated and virus-like messages are not easily removed, meaning they will remain in the network. The breadth and depth of the spread of these virus-like messages among groups determines the final degree of opinion polarization in group behavior. Obviously, under such a network structure, the polarization phenomenon is very easy to occur, and the polarization on Internet social platforms with similar network structures is also common; (2) unlike BA network, the small-world network is more similar to the actual interpersonal relationship network, because the 


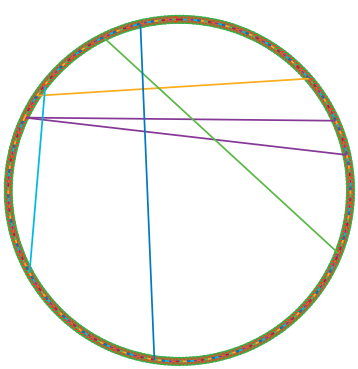

(a)

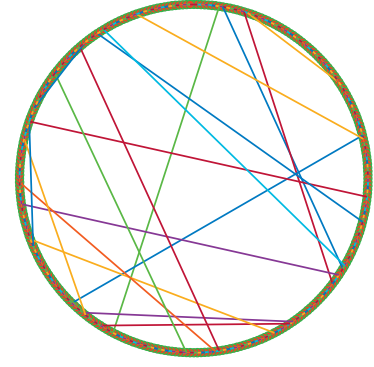

(b)

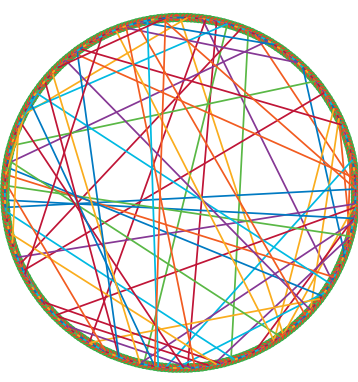

(c)

FIGURE 17: Small-world network $(p=0.01,0.05,0.1)$. (a) $p=0.01$, (b) $p=0.05$, and (c) $p=0.1$.

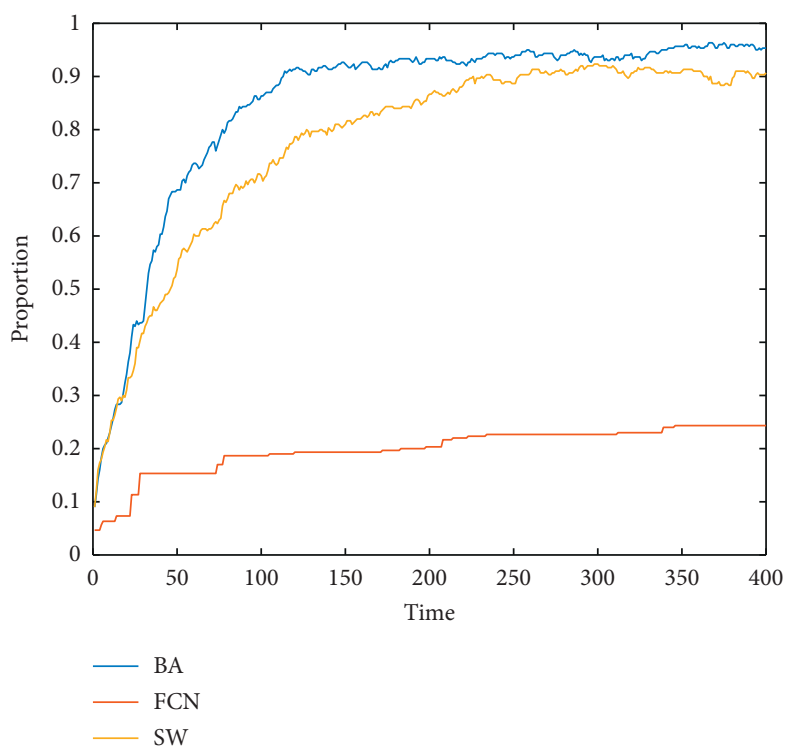

(a)

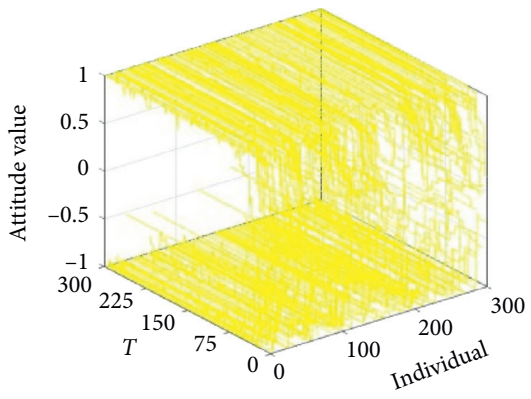

(c)

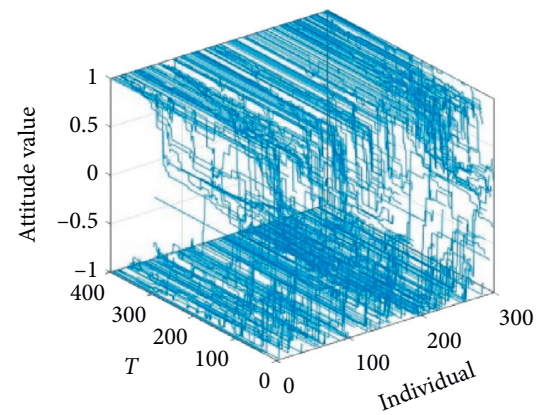

(b)

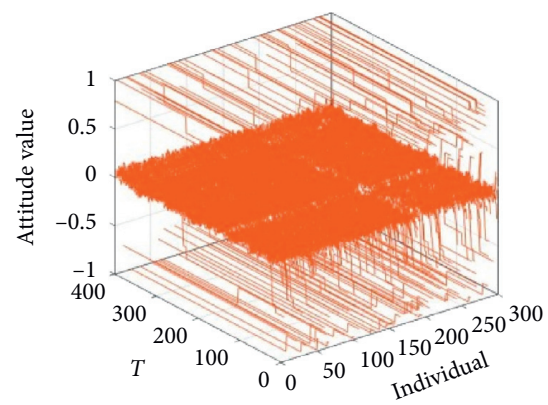

(d)

FIGURE 18: Comparison of attitude polarization process under different network structures $(p=0.1)$.

connection of most individuals is limited to the surrounding "neighbors". Under such network structure, the speed and breadth of information transmission depend on the "personality" possessed by the individual and the connection with the distant or higher-level individuals, which is equivalent to the reduction of the remote connection, that is, the reduction of individual contacts in the group. Thus, it will essentially affect the information transmission and reduce the interaction among individuals, forcing the group's opinions and attitudes to be less vivid and sharp; (3) in the fully connected network, each individual has a global perspective and can fully grasp the attitude values of other individuals. Under the condition of complete information symmetry, individuals tend to make rational decisions. As a result, the simulation results show that the polarization degree of individuals in the fully connected network is low, and the standard deviation is also small. With the deepening of interaction, the standard 


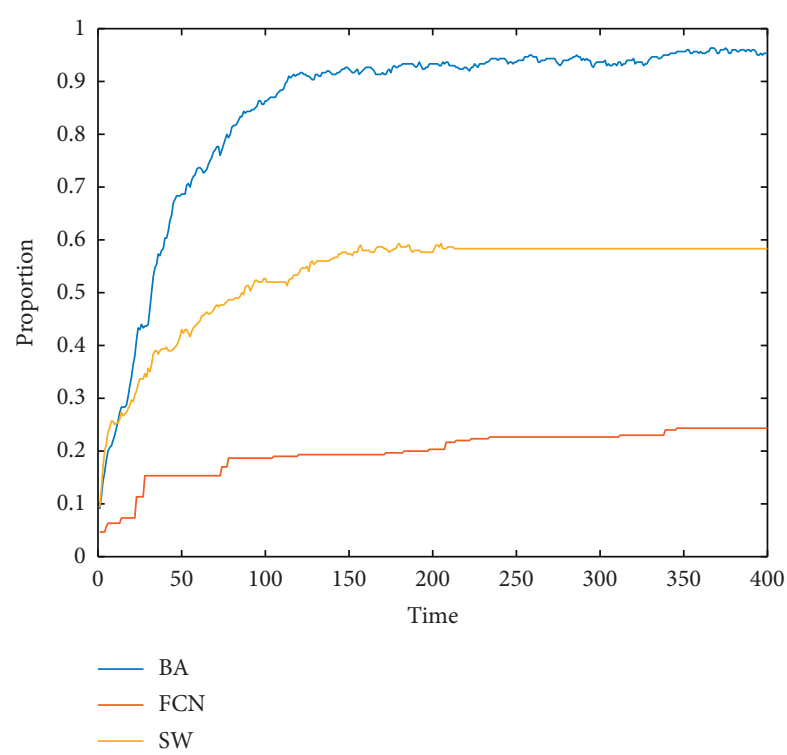

(a)

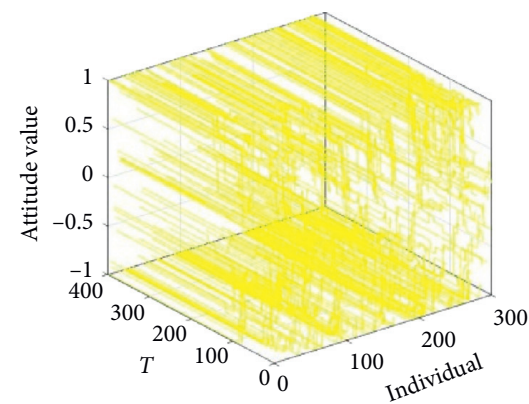

(c)

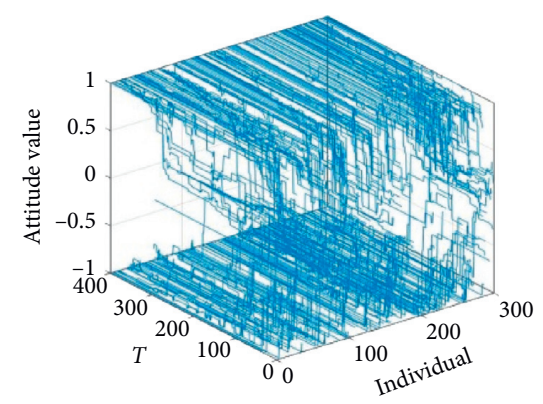

(b)

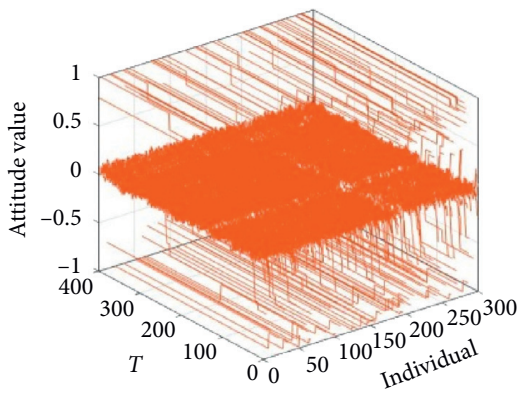

(d)

Figure 19: Comparison of attitude polarization process under different network structures $(p=0.05)$. Polarization trend charts of (a) participants under different network structures, (b) participants under BA network, (c) participants under SW network, and (d) participants under FCN network.

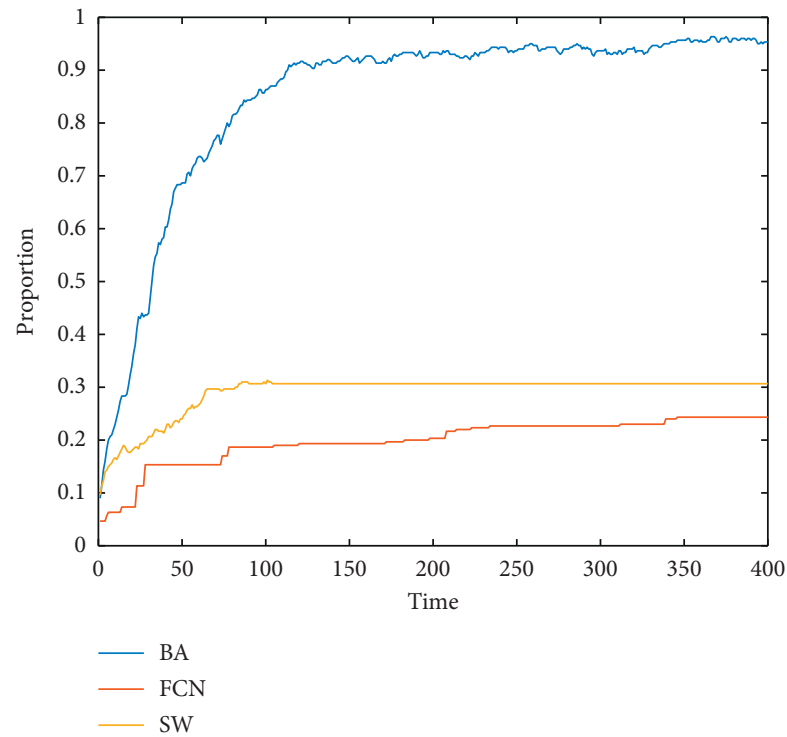

(a)

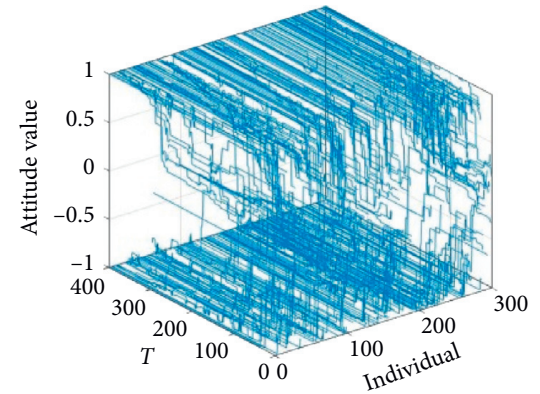

(b)

Figure 20: Continued. 


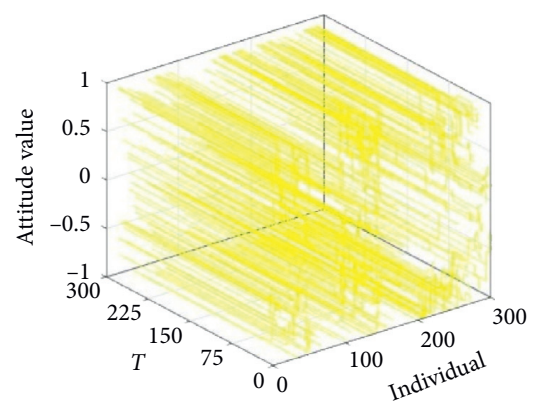

(c)

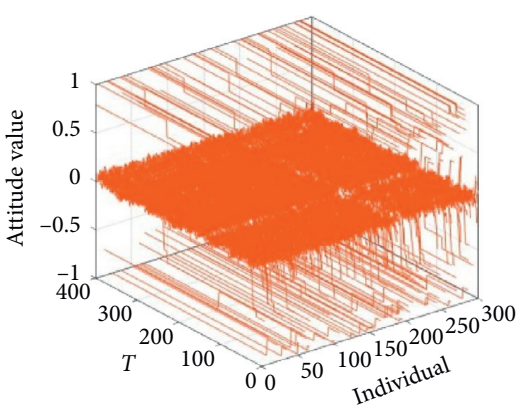

(d)

Figure 20: Comparison of attitude polarization process under different network structures $(p=0.01)$. Polarization trend charts of (a) participants under different network structures, (b) participants under BA network, (c) participants under SW network, and (d) participants under FCN network.

TABle 6: Average difference statistics of interaction stages at different times corresponding to Figures 18-20.

\begin{tabular}{lccccc}
\hline Network & & $T=1$ & $T=50$ & $T=100$ & $T=400$ \\
\hline BA & & 0.6138 & 0.8794 & 0.9352 & 0.9868 \\
\hline \multirow{3}{*}{ SW } & $p=0.1$ & 0.6138 & 0.8433 & 0.8947 & 0.9801 \\
& $p=0.05$ & 0.6138 & 0.773 & 0.8172 & 0.8563 \\
& $p=0.01$ & 0.6138 & 0.6893 & 0.727 & 0.7469 \\
\hline FCN & & 0.6138 & 0.5502 & 0.5424 & 0.5334 \\
\hline
\end{tabular}

deviation gradually decreases and the attitude values between individuals continue to assimilate.

All in all, an important reason for the endless stream of mass events and increasingly sharp contradictions in the network is that the structure of the social network is very easy to form the penetration and transmission of information, thus attracting individuals with different opinions. While in the process of further information dissemination, different groups develop their own opinions, and the conflict of ideas is aggravated in the process of communication, thus leading to polarization.

\section{Conclusions}

According to the polarization phenomenon in mass events, this paper combines the SIRS epidemic model with the polarization model and uses the multiagent Monte Carlo method to realize the evolution of agents' attitudes toward polarization. Also, through the analysis of the model parameters, this study analyzes the main factors that result in the polarization phenomenon. The main contributions and conclusions are illustrated as follows:

(1) This paper combines the SIRS epidemic model with the J-A model and regards the individual's attitude change affected by surrounding individuals as the infectious process of healthy individuals affected by infected ones. At the same time, the process of the infected people's immune relapse is metaphorized as the process in which an individual changes his attitude repeatedly, resulting in the quantitative accumulation to the qualitative change. The results obtained from simulation experiments further verify that the polarization effect is more significant with the integration of the SIRS epidemic model.

(2) The immune recovery rate $\gamma$ directly affects the overall progression of polarization. As the $\gamma$ value increases, the polarization phenomenon becomes more obvious. Therefore, when preventing or resolving mass events, it is possible to improve the individuals' immunity to public opinion information and effectively release authoritative speech from various channels, so that the audience may accurately distinguish the authenticity of the messages and thus reduce the $\gamma$ value to prevent the individuals from becoming communicators of false news and control extreme events or extreme public opinions.

(3) According to the relationship among individuals in the real society, this study assigns its different relationship strengths to reflect the influence of intimate individual opinions when making decisions. Based on this, this study explores the effect of changing the intensity of the relationship on the polarization process under the same conditions and finds that if there is an interest relationship among individuals in a group, the polarization of events or contradictions will be accelerated, and the final results will also be relatively intensified.

(4) Different network structures may affect the polarization process. The BA network may significantly lead to more obvious polarization than the small-world network, and the polarization process of small-world network is affected by the number of remote individuals. It shows that the average distances among individuals influence the polarization process.

However, because the development process of mass events is extremely complicated and the polarization phenomenon involves many factors and the real network structure is also complex [41], the theoretical discussions in this paper are not straightly applied to the empirical scene now. In future research, the role of opinion leaders [42], the nonbidirectional factors of interindividual relationship 
transmission [43], and individual's joining and leaving mechanism in the network [44], etc. should be considered as the research focus to make the model more realistic and the results of the simulation experiment more reasonable.

\section{Abbreviations}

SIRS: $\quad$ Susceptible-Infected-Recovered-Susceptible.

J-A model: Proposed by Jager and Amblard

BA Proposed by Barabasi and Albert

network:

D-W Proposed by Deffuant and Weisbuch

model:

SIR: $\quad$ Susceptible-Infected-Recovered

I2S2R: Infected-Susceptible-Infected-Susceptible-

Recovered

SEIR: $\quad$ Susceptible-Exposed-Infected-Resistant.

\section{Data Availability}

The data used to support the findings of this study are available from the corresponding author upon request.

\section{Conflicts of Interest}

The authors declare that they have no conflicts of interest.

\section{Authors' Contributions}

TC proposed the framework and wrote the whole manuscript. JS implemented the simulation experiments. JY, GC, and GL revised the manuscript. All authors read and approved the final manuscript.

\section{Acknowledgments}

This research was supported by the Major Project of $\mathrm{Hu}-$ manities and Social Sciences in University of Zhejiang Province, China (Grant no. 2018QN006), Hubei Key Laboratory of Mechanical Transmission and Manufacturing Engineering (2018A02) as well as Contemporary Business and Trade Research Center, and Center for Collaborative Innovation Studies of Modern Business of Zhejiang Gongshang University of China (Grant no. 14SMXY05YB).

\section{References}

[1] Y. F. Zhang, Emergent Computation of Collective Behavior in Social Systems, in Chinese, 2015.

[2] https://en.wikipedia.org/wiki/Killing_of_George_Floyd.

[3] https://www.kiro7.com/news/trending/george-floyd-demons trations-target-closes-down-175-stores-nationwide/RZJ524K UNJABXI23V63MPTXMZA/.

[4] https://www.nytimes.com/interactive/2020/07/03/us/georgefloyd-protests-crowd-size.html.

[5] T. Li, F. Zhang, H. Liu, and Y. Chen, "Threshold dynamics of an SIRS model with nonlinear incidence rate and transfer from infectious to susceptible," Applied Mathematics Letters, vol. 70, no. 1, pp. 52-57, 2017.

[6] W. Jager and F. d. r. Amblard, "Uniformity, bipolarization and pluriformity captured as generic stylized behavior with an agent-based simulation model of attitude change," Computational \& Mathematical Organization Theory, vol. 10, no. 4, pp. 295-303, 2005.

[7] Y. N. Kyrychko and K. B. Blyuss, "Global properties of a delayed SIR model with temporary immunity and nonlinear incidence rate," Nonlinear Analysis: Real World Applications, vol. 6, no. 3, pp. 495-507, 2005.

[8] J. Tanimoto, Fundamentals of Evolutionary Game Theory and its Applications, Vol. 10, Springer, Tokyo, Japan, 2015.

[9] Y. Zhang and J. Zhu, "Stability analysis ofI2S2Rrumor spreading model in complex networks," Physica A: Statistical Mechanics and Its Applications, vol. 503, pp. 862-881, 2018.

[10] L. A. Huo and N. Song, "Dynamical interplay between the dissemination of scientific knowledge and rumor spreading in emergency," Physica A: Statistical Mechanics and Its Applications, vol. 461, pp. 73-84, 2016.

[11] J. Wu and R. Gergely, "SEIR epidemiological model with varying infectivity and infinite delay," Mathematical Biosciences and Engineering, vol. 5, no. 2, pp. 389-402, 2008.

[12] T. Chen, J. Shi, J. Yang, and G. Li, "Enhancing network cluster synchronization capability based on artificial immune algorithm[J]," Human-centric Computing and Information Sciences, vol. 9, p. 3, 2019.

[13] N. Boccara and K. Cheong, "Automata network SIR models for the spread of infectious diseases in populations of moving individuals," Journal of Physics A: Mathematical and General, vol. 25, no. 9, pp. 2447-2461, 1992.

[14] K. M. A. Kabir, K. Kuga, and J. Tanimoto, "Analysis of SIR epidemic model with information spreading of awareness," Chaos, Solitons \& Fractals, vol. 119, pp. 118-125, 2019.

[15] K. M. A. Kabir and J. Tanimoto, "Analysis of epidemic outbreaks in two-layer networks with different structures for information spreading and disease diffusion," Communications in Nonlinear Science and Numerical Simulation, vol. 72, pp. 565-574, 2019.

[16] K. M. A. Kabir, K. Kuga, and J. Tanimoto, "Effect of Information spreading to suppress the disease contagion on the epidemic vaccination game," Chaos, Solitons \& Fractals, vol. 119, pp. 180-187, 2019.

[17] K. M. A. Kabir, K. Kuga, and J. Tanimoto, "The impact of information spreading on epidemic vaccination game dynamics in a heterogeneous complex network- A theoretical approach. Chaos," Solitons \& Fractals, vol. 132, 2020.

[18] K. M. A. Kabir and J. Tanimoto, "Vaccination strategies in a two-layer SIR/V-UA epidemic model with costly information and buzz effect," Communications in Nonlinear Science and Numerical Simulation, vol. 76, pp. 92-108, 2019.

[19] X. Y. Lan and Y. X. Xia, "Research on the evolution mechanism and trend prediction of netizens for public opinion big data," Journal of Intelligence, vol. 36, no. 11, pp. 134-140, 2017, in Chinese.

[20] G. Deffuant, N. David, and F. Amblard, "Mixing beliefs among interacting agents," Advances in Complex Systems, vol. 3, no. 1-4, pp. 87-98, 2000.

[21] G. Weisbuch, G. Deffuant, and F. Amblard, "Meet, discuss, and segregate!" Complexity, vol. 7, no. 3, pp. 55-63, 2010.

[22] T. Chen, Q. Li, P. Fu et al., "Public opinion polarization by individual revenue from the social preference theory," International Journal of Environmental Research and Public Health, vol. 17, no. 3, p. 946, 2020.

[23] T. Chen, Y. Wang, J. Yang, and G. Cong, "Modeling public opinion reversal process with the considerations of external intervention information and individual internal characteristics," Healthcare, vol. 8, no. 2, p. 160, 2020. 
[24] P. Siedlecki, J. Szwabiński, and T. Weron, "The interplay between conformity and anticonformity and its polarizing effect on society[J]," Journal of Artificial Societies \& Social Simulation, vol. 19, no. 4, 2016.

[25] K. Tyll and T. W. SzwabińskiJanusz, "Conformity, anticonformity and polarization of opinions: insights from a mathematical model of opinion dynamics," Entropy, vol. 19, no. 7 , p. 371,2017

[26] A. Flache, M. Mäs, T. Feliciani et al., "Models of social influence: towards the next frontiers," Journal of Artificial Societies and Social Simulation, vol. 20, no. 4, p. 2, 2017.

[27] J. Li and R. Xiao, "Agent-based modelling approach for multidimensional opinion polarization in collective behaviour[J]," Journal of Artificial Societies \& Social Simulation, vol. 20, no. 2, p. 14, 2017.

[28] A. Bramson, P. Grim, D. J. Singer et al., "Disambiguation of social polarization concepts and measures," The Journal of Mathematical Sociology, vol. 40, no. 2, pp. 80-111, 2016.

[29] A. Bramson, P. Grim, D. J. Singer et al., "Understanding polarization: meanings, measures, and model evaluation," Philosophy of Science, vol. 84, no. 1, pp. 115-159, 2017.

[30] S. Wang, X. Zhu, and X. Pan, "Research on the mechanism for group polarization in online public opinion dynamics[J]," Journal of the China Society for Scientific \& Technical Information, vol. 33, no. 6, pp. 614-622, 2014, in Chinese.

[31] T. Chen, Q. Li, J. Yang, G. Cong, and G. Li, "Modeling of the public opinion polarization process with the considerations of individual heterogeneity and dynamic conformity," Mathematics, vol. 7, no. 10, p. 917, 2019.

[32] A. Sîrbu, V. Loreto, V. D. P. Servedio, and F. Tria, "Opinion dynamics with disagreement and modulated information[J]," Journal of Statistical Physics, vol. 151, no. 1-2, pp. 218-237, 2013.

[33] Y. Zhang, Y. Wang, T. Chen, and J. Shi, "Agent-based modeling approach for group polarization behavior considering conformity and network relationship strength[J]," Concurrency And Computation-Practice \& Experience, vol. 32, no. 14, Article ID e5707, 2020.

[34] M. Stella, E. Ferrara, and M. De Domenico, "Bots increase exposure to negative and inflammatory content in online social systems," Proceedings of the National Academy of Sciences, vol. 115, no. 49, pp. 12435-12440, 2019.

[35] M. D. Conover, B. Goncalves, J. Ratkiewicz, A. Flammini, and F. Menczer, "Predicting the political alignment of twitter users," in Proceedings of the 2011 IEEE Third International Conference on Privacy, Security, Risk and Trust and 2011 IEEE Third International Conference on Social Computing, October 2011.

[36] F. Baumann, P. Lorenz-Spreen, I. M. Sokolov, and M. Starnini, "Modeling echo chambers and polarization dynamics in social networks," 2019, http://arxiv.org/abs/1906. 12325.

[37] Y. Gong and S. Liang, "Agent-based modeling and simulation of group polarization formation on group-buying of new technological product," Science and Technology Management Research, vol. 35, no. 18, pp. 201-207, 2015, in Chinese.

[38] C. W. Seagren, "A replication and analysis of tiebout competition using an agent-based computational model," Social Science Computer Review, vol. 33, no. 2, pp. 198-216, 2014.

[39] https://money.cnn.com/2017/09/07/technology/business/equ ifax-data-breach/index.html.

[40] https://en.wikipedia.org/wiki/2017_Equifax_data_breach.

[41] T. Chen, L. Peng, X. Yin, J. Rong, J. Yang, and G. Cong, "Analysis of user satisfaction with online education platforms in China during the COVID-19 pandemic," Healthcare, vol. 8 , no. 3, p. 200, 2020.

[42] C. Xu, "A novel recommendation method based on social network using matrix factorization technique," Information Processing \& Management, vol. 54, no. 3, pp. 463-474, 2018.

[43] T. Chen, S. Wu, J. Yang, G. Cong, and G. Li, "Modeling of reliable emergency supply scheduling and its solution based on artificial bee colony algorithm under road-varying network after sudden-onset disasters," Complexity, vol. 2020, Article ID 7501891, 15 pages, 2020.

[44] T. Chen, S. Wu, J. Yang, and G. Cong, "Risk propagation model and its simulation of emergency logistics network based on material reliability," International Journal of Environmental Research and Public Health, vol. 16, no. 23, p. 4677, 2019. 